\title{
Quantifying the effects of higher order coupling terms on fits using a second order Jahn-Teller Hamiltonian
}

\author{
Henry K. Tran ${ }^{a}$, John F. Stanton ${ }^{b}$, Terry A. Miller ${ }^{a}$ \\ ${ }^{a}$ Department of Chemistry, The Ohio State University, \\ 100 W. $18^{\text {th }}$ Avenue \\ Columbus Ohio 43210, USA \\ email: miller.104@osu.edu \\ ${ }^{b}$ Quantum Theory Project, Departments of Chemistry and Physics \\ University of Florida, Gainesville, Florida 32611, USA
}

\begin{abstract}
The limitations associated with the common practice of fitting a quadratic Hamiltonian to vibronic levels of a Jahn-Teller system have been explored quantitatively. Satisfactory results for the prototypical $\tilde{X}^{2} E^{\prime}$ state of $\mathrm{Li}_{3}$ are obtained from fits to both experimental spectral data and to an "artificial" spectrum calculated by a quartic Hamiltonian which accurately reproduces the adiabatic potential obtained from state-of-the-art quantum chemistry calculations. However the values of the Jahn-Teller parameters, stabilization energy, and pseudo-rotation barrier obtained from the quadratic fit differ markedly from those associated with the $a b$ initio potential. Nonetheless the RMS deviations of the fits are not strikingly different. Guidelines are suggested for comparing parameters obtained from fits to experiment to those obtained by direct calculation, but a principal conclusion of this work is that such comparisons must be done with a high degree of caution.
\end{abstract}

\section{Introduction}

In 1936, Jahn and Teller published their seminal paper on the spontaneous symmetry breaking of molecules in degenerate electronic states, which has since become known as the Jahn-Teller (JT) effect.[1] Eight decades later, multiple experimental techniques exist to allow for the detection of this effect in isolated molecules.[2] Similarly, theoretical techniques 
have advanced; accurate $a b$ initio calculations can now be done on systems exhibiting the JT effect. One may wonder what is left to discuss after all this time. However, despite nearly a century of advancement, there is still much to be learned about the analyses of the spectra of JT active molecules. One recurring problem, which forms the focus of this work, is why experimental and theoretical analyses of the JT effect often disagree by more than what one might expect.

Even though the capabilities of both experimental and theoretical analyses have greatly expanded, both still have their shortcomings. Approximations must be made in both areas to obtain molecular parameters, and these carry an inherent error giving rise to a multitude of questions regarding the validity of experimental and theoretical analyses, as well as to how they complement each other. In what cases do the typical approximations jeopardize the analysis? How can we assess the quality of an analysis? If we cannot avoid an approximation, then how does that approximation bias the results? These are critical questions that should not be ignored. The answers can either validate or invalidate important conclusions, and point to important issues that must be addressed. In the midst of burgeoning experimental developments and accelerating computational technology, it is worthwhile to consider some of these questions.

On the experimental side, techniques have improved to the point where we can observe characteristic patterns in spectral data resulting from the JT effect, but a difficulty lies in extracting physically meaningful quantities from this information. To gain insight into the relevant potential energy surfaces (PES), experimental analyses rely on representing the PES by an analytical form containing parameters that are fit to observation. For the JT effect, the analytical potential is typically represented by a (truncated) Taylor expansion and the coefficients in the expansion are fit to experimental data.[2] However, with enough parameters, any model can accurately reproduce line positions. Given the limited number of lines present in a typical vibronic spectrum, a Taylor expansion for the PES of a JT active molecule must be aggressively truncated to second order, or even a subset thereof, to obtain 
a useful fit. There are obvious concerns associated with this approach. In what cases is the quadratic expansion sufficient and how can we identify situations where it is not? How are results from the fit changed from the actual values if the true potential contains important higher order terms?

The theoretical analyses are also not free from limitations. First, the conical intersection that is the result of the JT effect invalidates the Born-Oppenheimer approximation, greatly complicating electronic structure calculations; one needs to use methods that provide a balanced treatment of more than one electronic state, such as multireference approaches or equation-of-motion theory.[3] Obviously, finite basis set and electron correlation errors must also be taken into account, as well. This paper will not focus on issues limiting the accuracy of electronic structure calculations. Rather, it will be concerned with assessing and connecting state-of-the-art theoretical and experimental methods for elucidating properties of the PES for JT-active molecules.

Such comparison between experimental and theoretical analyses has been a topic of longstanding effort. We briefly review some examples of JT active molecules that have been analyzed with both approaches. The JT effect in the ground states of halogenated benzene cations[4] has been studied in our group. The spectrum of organic radicals $\mathrm{C}_{6} \mathrm{H}_{3} \mathrm{~F}_{3}^{+}$ and $\mathrm{C}_{6} \mathrm{H}_{3} \mathrm{Cl}_{3}^{+}$were collected[5] and analyzed using primarily a linear treatment of the JT interaction. [6] The spectrum of the hexa-substituted benzene, $\mathrm{C}_{6} \mathrm{~F}_{6}^{+}$, was obtained in matrix experiments and analyzed using the same simple model.[7] The geometry was determined and the JT distortion found to be weak. Later theoretical predictions on $\mathrm{C}_{6} \mathrm{~F}_{6}^{+}$were made using MP2 and CISD electronic structure methods.[8] While the total Jahn-Teller stabilization energy (JTSE) was found to be similar to that inferred from experiment, there was a large discrepancy in the $\mathrm{C}-\mathrm{C}$ stretching frequency as well as $\mathrm{C}-\mathrm{C}$ bond length, and in the strength of the JT effect in individual modes. The theory typically estimated a larger JT effect than that obtained by fits to the experimental data.

The ground state of the cyclopentadienyl radical, $\tilde{X}^{2} E_{1}{ }^{\prime \prime}$, is subject to the JT effect. A 
large number of experimental[9, 10, 11, 12, 13] and theoretical[14, 15, 16, 17, 17, 18] studies have been reported on this molecule. An experimental analysis was conducted by Purins and Feeley[9] in which they determined that the JT effect should be significant. Engelking and Lineberger[10] also observed the spectrum by laser photodetachment spectroscopy of the anion and analyzed the results for the JT effect. Contrary to Purins and Feeley, they believed the JT effect to be relatively weak. Laser-induced fluorescence (LIF) techniques have also been applied to the radical by Nelson et al.[11] They found that the magnitude of the JT effect was somewhere between that found in the previously mentioned studies. LIF has also been used in our group to observe this molecule[12] and its deuterated species.[13] The analysis revealed a significant JT effect similar to that reported by Purins and Feeley. A later analysis (based on a quadratic model in our group) of the laser excited, vibrationally resolved emission from the $\tilde{A}^{2} A_{2}^{\prime \prime}-\tilde{X}^{2} E_{1}^{\prime \prime}$ electronic transition found[19] a JTSE of 1237 $\mathrm{cm}^{-1}$.

One of the first ab initio calculations by Liehr[14] found a JTSE of $560 \mathrm{~cm}^{-1}$, and no pseudo-rotation barrier, the latter being unsurprising since the second-order JT coupling along the active $e_{2}^{\prime}$ modes (which gives rise to distinct minima and maxima along the pseudorotation coordinate) vanishes by symmetry in this case. In a more recent theoretical analysis[18], the JTSE at the CCSD(T)/cc-pVTZ level of theory was found to be $2273 \mathrm{~cm}^{-1}$, close to the value determined by Borden and Davidson.[20] A later ab initio calculation in our group[21] using CAS(5,5)/cc-pVDZ level of theory deduced a similar JTSE of 2139 $\mathrm{cm}^{-1}$ and a negligible pseudorotation barrier of about $4 \mathrm{~cm}^{-1}$. A later study published by Ichino et al.[22] analyzed the ground state of the cyclopentadienyl radical using experimental techniques and theoretical calculations at the EOMIP-CCSD level of theory, but with a relatively small basis set. They included linear and bilinear JT coupling terms in the PES and found a more modest JTSE of $1582 \mathrm{~cm}^{-1}$. Even given these ranges of values, all of the recent theoretical results predict a stronger JT effect than deduced in any of the experimental analyses. 
The methoxy radical family $\left(\mathrm{CH}_{3} \mathrm{O}, \mathrm{CH}_{3} \mathrm{~S}, \mathrm{CF}_{3} \mathrm{O}, \mathrm{CF}_{3} \mathrm{~S}\right)$ has been the subject of many high-resolution experimental and theoretical studies. They all have a JT active ground state, $\tilde{X}^{2} E$. $\mathrm{CH}_{3} \mathrm{O}$ in particular is the best-studied JT radical and arguably the most extensively studied polyatomic radical of any kind. As summarized in the review by Barckholtz and Miller[2], a wide range of experiments has been conducted on methoxy including emission, [23, 24] LIF of the excited states[25, 26, 27, 28] and ground state, [28, 29, 30] $\mathrm{SEP},[31,32,33]$ electron spin resonance, [34] photoelectron spectroscopy, [35, 36] and fast beam photofragmentation of the excited state.[37, 38] Rotationally resolved spectra have been obtained by a number of techniques including far-infrared laser magnetic resonance (LMR),[39, 40] microwave studies,[41, 42, 43] and LIF.[44, 45, 46] Many of these experiments have also been applied to the deuterated species.[24, 28, 47, 38] An experimental data fit yields a JTSE of $419 \mathrm{~cm}^{-1}$.[2] There have also been numerous ab initio analyses on methoxy.[48, 49, 50, 51, 52, 53, 54] Many body perturbation theory estimates the JTSE of the ground electronic state to be $224 \mathrm{~cm}^{-1}$ and also determines the spin-orbit coupling.[48] For this same state, EOMIP-CCSD calculations estimate a JTSE of $270 \mathrm{~cm}^{-1}$ and a pseudorotation barrier of $49 \mathrm{~cm}^{-1} \cdot[51]$

Issues associated with experimental v. theoretical characterization of the JT effect have reemerged in our recent work[55] on the well known[56, 57, 58] atmospheric oxident and intermediate, $\mathrm{NO}_{3}$. There is expected to be significant pseudo-JT coupling between the $\widetilde{X}^{2} A_{2}^{\prime}, \widetilde{A}^{2} E^{\prime \prime}$, and $\widetilde{B}^{2} E^{\prime}$ states, and JT coupling within the $\tilde{A}$ and $\tilde{B}$ states. Much of the experimental $[59,60,61,62,63,64,65,66]$ and theoretical[67, 68, 69] analyses in the past three decades have returned contradictory results on the magnitude of the JT coupling in these two degenerate states. Most recently, the $\widetilde{A}^{2} E^{\prime \prime}$ state of $\mathrm{NO}_{3}$ has been analyzed using both experimental and theoretical techniques.[55] In that work, an experimental fit of the vibronic levels in the $\widetilde{A}^{2} E^{\prime \prime} \leftarrow \widetilde{X}^{2} A_{2}^{\prime}$ spectrum was conducted as well as a theoretical calculation using EOMIP-CCSDT/ANO0 for the $\widetilde{A}^{2} E^{\prime \prime}$ state. The latter generally agrees with an independent calculation using MRCI[70], both of which indicate a significant JT 
effect. The magnitude of the JT effect estimated from experimental results[55] was even greater than that predicted by theory. In spite of that, early experimental work[71] on the rotational structure of $4_{0}^{1}$ band concluded that no JT effect was necessary to explain the observed spectra.

$\mathrm{Li}_{3}$ is an example of a "simple" JT active molecule (there is but a single degenerate mode, and only one non-degenerate mode), exhibiting the ubiquitous $E \otimes e$ JT effect in its $\tilde{X}^{2} E^{\prime}$ state. Although a small system, the JT coupling in the $\tilde{X}^{2} E^{\prime}$ state of $\mathrm{Li}_{3}$ is still something of an open problem. Gerber and Schumacher were among the first to propose that the structure of $\mathrm{Li}_{3}$ under the $\mathrm{JT}$ effect would be a $\mathrm{C}_{2 v}$ isosceles triangle.[72] In that paper, they utilized the coupled electron pair approximation (CEPA). Later, Martins et al. calculated the electronic structure of the $\tilde{X}^{2} E^{\prime}$ state using density functional theory.[73] Their results also support the $\mathrm{C}_{2 v}$ geometry. Thompson et al. devised a model potential to fit these ab initio calculations. [74] From the calculation of Gerber and Schumacher,[72] a JTSE of $435 \mathrm{~cm}^{-1}$ and pseudo-rotation barrier of $37 \mathrm{~cm}^{-1}$ were determined. From the calculation of Martins et al.,[73] a JTSE of $449 \mathrm{~cm}^{-1}$ and pseudo-rotation barrier of $5 \mathrm{~cm}^{-1}$ were determined. Extensive MRCI calculations have been conducted on the $\tilde{X}^{2} E^{\prime}$ state[75, 76] as well as the $A^{2} E^{\prime \prime}$ state.[77] Ehara and Yamashita[75] found values of $506 \mathrm{~cm}^{-1}$ and $73.5 \mathrm{~cm}^{-1}$ for the JTSE and pseudo-rotation barrier in the $\tilde{X}^{2} E^{\prime}$ state. These agree essentially quantitatively with calculated values of $504 \mathrm{~cm}^{-1}$ and $77 \mathrm{~cm}^{-1}$ from Larson and collaborators.[76] Experimental vibronic levels in the $\tilde{X}^{2} E^{\prime}$ state of $\mathrm{Li}_{3}$ have been observed[78, 79] as well and analyzed using a truncated quadratic potential function. [78] The latter indicates that the JTSE is $256 \mathrm{~cm}^{-1}$, significantly less than obtained in calculations, and the pseudo-rotation barrier is $19 \mathrm{~cm}^{-1}$, which is also below calculated values. The work of Krämer[79] in particular also documented extensive and highly sophisticated quantum chemical calculations.

While not an exhaustive survey, the results discussed above establish that there are systematic inconsistencies between the conclusions of experimental spectral analyses and quantum chemistry calculations for JT-active molecules. As mentioned earlier, there are 
known limitations to both the experimental and computational approaches, but often the resulting discrepancies seem greater than one would expect for the known limitations. The purpose of this paper is to explore critical results for JT-active molecules from theoretical computations and experimental observations. For this comparison, we choose the last example discussed above, the $\tilde{X}^{2} E^{\prime}$ state of $\mathrm{Li}_{3}$. The motivation for this choice is that it is a light, triatomic molecule in its ground state, for which modern quantum chemistry calculations should be highly accurate. Indeed our new calculation of its PES at the EOMEA-CCSDT level theory is in close agreement with previous calculations of Refs. [75, 76, 79], and we use this approach to parametrize a Hamiltonian[80] truncated at fourth order. As described in more detail below, we scale the cubic and quartic terms of the potential with factors ranging from zero to unity to quantify the effect of higher order terms on the fit values of the JT parameters when a truncated quadratic model is used.

Experimentally $\mathrm{Li}_{3}$ is not the best characterized JT-active molecule. (Note that while the experimental assignments for this state are arguably far from secure, much of the work ahead will focus on using the "experimental approach" - fitting levels to a truncated Hamiltonian with calculated levels, for which assignments are correct by construction.) However, it is well enough characterized for the purpose of demonstrating that both our theoretical approach and computed potential have physical significance. We are able to predict vibronic energy levels of ground state $\mathrm{Li}_{3}$ that are close to experimentally measured band positions. We compare the experimental level positions to predictions from both a quadratic and quartic parameterization of the potential as well as predictions from a quadratic JT Hamiltonian with its parameters best fit, in a least-squares sense, to the experimental levels. These comparisons clearly reveal potential issues that may arise when comparing quantum chemistry computational results with experimental observations for JT-active molecules. 


\section{Theory}

The JT effect serves to break electronic degeneracy and some experimental analyses rely on treatment of the electronic states adiabatically. The experimental fit model and theory to be introduced later treat the electronic states "quasidiabatically," which we shall refer to as the diabatic treatment for brevity. An overview of both approaches is given in this section, but ultimately the approaches are equivalent if carried out rigorously and to a consistent order.

The full molecular Hamiltonian neglecting spin, relativistic, and other higher order effects is

$$
\begin{aligned}
\hat{\mathcal{H}} & =\hat{T}_{N}+\hat{T}_{e}+\hat{V}_{n e}+\hat{V}_{e e}+\hat{V}_{n n} \\
& =\hat{T}_{N}+\hat{\mathcal{H}}_{e}
\end{aligned}
$$

where the terms from left to right are: nuclear kinetic energy associated with only vibrations for the present application, electron kinetic energy, nuclear-electron attraction, electronelectron repulsion, and nuclear-nuclear repulsion. The last four terms form the electronic Hamiltonian $\hat{\mathcal{H}}_{e}$, which can in principle be diagonalized at any geometry $\mathbf{R}$ to give adiabatic electronic eigenvalues $V_{i}(\mathbf{R})$ and eigenfunctions $\psi_{i}(\mathbf{r} ; \mathbf{R})$ where $\mathbf{r}$ and $\mathbf{R}$ denote the set of electron and nuclear coordinates respectively. Correspondingly, the eigenfunctions of the

full Hamiltonian, $\hat{\mathcal{H}}$, will be denoted $\Psi_{k}(\mathbf{r}, \mathbf{R})$. In the following subsections, we review and relate various theoretical and experimental approaches employed to quantify the JT effect in molecules.

\subsection{Adiabatic Treatment}

The adiabatic treatment is well-known. The basis functions, $\Psi_{k}(\mathbf{r}, \mathbf{R})$, can be written

$$
\Psi_{k}(\mathbf{r}, \mathbf{R})=\chi_{j_{k}}(\mathbf{R}) \psi_{i_{k}}(\mathbf{r} ; \mathbf{R})
$$


where $\chi_{j_{k}}(\mathbf{R})$ are vibrational (or "rovibrational" if rotational motion were to be considered) basis functions. The matrix of Equation $\hat{\mathcal{H}}$, written in this basis is

$$
\hat{\mathcal{H}}=\left(\begin{array}{ccc}
\mathcal{T}_{N}^{(A, A)} & \mathcal{T}_{N}^{(A, B)} & \ldots \\
\mathcal{T}_{N}^{(B, A)} & \mathcal{T}_{N}^{(B, B)} & \ldots \\
\vdots & \vdots & \ddots
\end{array}\right)+\left(\begin{array}{ccc}
V_{1}(\mathbf{R}) \mathbb{1} & 0 & \ldots \\
0 & V_{2}(\mathbf{R}) \mathbb{1} & \ldots \\
\vdots & \vdots & \ddots
\end{array}\right)
$$

where $\mathcal{T}_{N}^{\left(i, i^{\prime}\right)}$ is the matrix with elements $\left\langle\chi_{j}\left|\left\langle\psi_{i}\left|\hat{T}_{N}\right| \psi_{i^{\prime}}\right\rangle\right| \chi_{j^{\prime}}\right\rangle$ and $\mathbb{1}$ is the identity matrix of the dimension of the vibrational basis. The two components of the degenerate electronic state are denoted here by $A$ and $B$. Within the Born-Oppenheimer approximation, the kinetic coupling terms $\mathcal{T}_{N}^{(i, j)}$ with $i \neq j$ are ignored and the $\hat{\mathcal{H}}$ matrix is diagonal in the electronic basis in this picture. However, in a JT case, this kinetic coupling is pathologically large and cannot be neglected.

\subsection{Diabatic Treatment}

The formulation presented here briefly summarizes the diabatic treatment of Köppel et al.[80] Starting from the Born-Huang expansion, the rigorously exact vibronic eigenfunction can be expressed

$$
\Psi_{k}(\mathbf{r}, \mathbf{R})=\sum_{i j} C_{i j ; k} \chi_{j}(\mathbf{R}) \psi_{i}\left(\mathbf{r} ; \mathbf{R}_{0}\right)
$$

The electronic basis functions here have only a weak dependence on $\mathbf{R}$, and simply "follow the nuclei" from the reference geometry $\mathbf{R}_{0}$. The form of the Hamiltonian in the basis of product states $\chi_{j}(\mathbf{R}) \psi_{i}\left(\mathbf{r} ; \mathbf{R}_{0}\right)$ is

$$
\hat{\mathcal{H}}=\left(\begin{array}{ccc}
\mathcal{T}_{N} & 0 & \cdots \\
0 & \mathcal{T}_{N} & \cdots \\
\vdots & \vdots & \ddots
\end{array}\right)+\left(\begin{array}{ccc}
V_{A A}(\mathbf{R}) & V_{A B}(\mathbf{R}) & \ldots \\
V_{B A}(\mathbf{R}) & V_{B B}(\mathbf{R}) & \ldots \\
\vdots & \vdots & \ddots
\end{array}\right)
$$


$\mathcal{T}_{N}$ are matrices with elements $\left\langle\chi_{j}\left|\hat{T}_{N}\right| \chi_{j^{\prime}}\right\rangle . \hat{T}_{N}$ is assumed to be diagonal in the electronic part of the basis, with the coupling between states now transferred to the potential. The $V_{\alpha \beta}(R)$ are matrices with elements $V_{i i^{\prime}, j j^{\prime}}=\left\langle\chi_{j}\left|\left\langle\psi_{i}\left|\hat{\mathcal{H}}_{e}\right| \psi_{i^{\prime}}\right\rangle\right| \chi_{j^{\prime}}\right\rangle$, which contain both the diagonal diabatic electronic potentials, $V_{i i}$, and the terms, $V_{i i^{\prime}}$, that couple the diabatic states.

\section{Methodology}

In this section, we first discuss methods for characterizing the JT effect from observed level energies from spectra. Second, we provide an overview of methods for calculating the vibronic spectrum of JT active molecules from first principles. The explicit Hamiltonian will be discussed in both of these cases. Third, a scheme is presented to evaluate the impact of truncating the potential used in "experimental analyses". In Section 4, we will use these methods to analyze the JT effect in the $\tilde{X}^{2} E^{\prime}$ state $\mathrm{Li}_{3}$.

The starting point for all these analyses is Equation (6) whose last term forms the matrix of the potential energy operator, $\hat{V}$, of $\hat{\mathcal{H}}_{e}$. In order to calculate vibronic spectra from $\hat{\mathcal{H}}, \hat{V}$ is typically expanded as a Taylor series in normal mode coordinates, $q_{k}$.

$$
\hat{V}=\sum_{n=0}^{\infty}\left[\left.\sum_{k_{1}+\cdots+k_{p}=n}\left(\frac{1}{k_{1} ! \ldots k_{p} !}\right)\left(\frac{\partial^{k_{1}+\cdots+k_{p}} \hat{V}}{\partial q_{1}^{k_{1}} \cdots \partial q_{p}^{k_{p}}}\right)\right|_{\mathbf{R}_{0}} q_{1}^{k_{1}} \cdots q_{p}^{k_{p}}\right]=\sum_{n^{\prime}=2,4 \cdots} \hat{V}^{\left(n^{\prime}\right)}
$$

where the last term $n^{\prime}$ is defined such that it is summed over even integers and includes both the $n^{\prime}=n$ and $n^{\prime}=n-1$ terms. For the JT cases considered here, the $n^{\prime}=0$ term is dropped because it is a constant that can be set to zero for diagonal terms and not included for off-diagonal terms since the states do not interact at the reference geometry, $\mathrm{R}_{0}$. For the $\mathrm{JT}$ calculation, we take the reference geometry $\mathrm{R}_{0}$ to be the conical intersection minimum and $p$ is the number of normal modes. 
Using this expansion we can write the $n^{\prime}$ order JT Hamiltonian as

$$
\mathcal{H}_{J T}^{\left(n^{\prime}\right)}=T_{N}+\hat{V}^{(2)}+\hat{V}^{(4)}+\cdots+\hat{V}^{\left(n^{\prime}\right)}
$$

If the expansion is carried out to infinite order the Hamiltonian should be exact, but of course this is not feasible either computationally or experimentally. If we truncate the expansion at $n^{\prime}=2$, we have the quadratic JT Hamiltonian $\mathcal{H}_{J T}^{(2)}$, at $n^{\prime}=4$, the quartic JT Hamiltonian, $\mathcal{H}_{J T}^{(4)}$, and so on.

\section{1. "Experimental" Methodology}

\subsubsection{Explicit Vibronic Hamiltonian}

Experimentally based analyses often seek to determine the coefficients of the potential expansion truncated at $n^{\prime}=2$, by fitting differences in eigenvalues of $\mathcal{H}_{J T}^{(2)}$ to observed levels in experimental spectra. Using dimensionless normal coordinates $(q)$, in the diabatic electronic basis, $\left|\psi_{i}\right\rangle$, the matrix elements of the potential have the form

$$
\left\langle\psi^{i}\left|\hat{V}^{(2)}\right| \psi^{i^{\prime}}\right\rangle=\sum_{k} F_{k}^{i i^{\prime}} q_{k}+\frac{1}{2} \sum_{k, l} F_{k l}^{i i^{\prime}} q_{k} q_{l}
$$

where the coefficient $F$ can generally be defined by

$$
F_{k \ldots l}^{i i^{\prime}}=\frac{\partial}{\partial q_{k}}\left(\cdots\left(\frac{\partial}{\partial q_{l}}\left\langle\psi^{i}|\hat{V}| \psi^{i^{\prime}}\right\rangle\right) \cdots\right)
$$

For $\mathrm{Li}_{3}$, the sums run over the totally symmetric reduced normal coordinate $q_{1}$ as well as the two (real and Cartesian) components of the degenerate mode $q_{2}$, which can be chosen to be those which transform as $a_{1}$ (denoted as $q_{2 a}$ ) and $b_{2}$ (denoted as $q_{2 b}$ ) in the $C_{2 v}$ subgroup of the $D_{3 h}$ molecular point group. The ground state of $\mathrm{Li}_{3}$ is ${ }^{2} E^{\prime}$ whose components are labeled $\mathrm{A}$ and $\mathrm{B}$; it has a degenerate, asymmetric-bending $e^{\prime}$ vibration, labeled 2, whose 
fundamental level splits into $a_{1}^{\prime}+a_{2}^{\prime}+e^{\prime}$ vibronic components. It also has a totally symmetric stretch $a_{1}^{\prime}$ vibration, labeled 1 , whose fundamental level of course has $e^{\prime}$ vibronic symmetry.

Explicitly we can write out the non-zero matrix elements of $V^{(2)}$ in Equation (9),

$$
\begin{aligned}
\left\langle\psi^{A}\left|V^{(2)}\right| \psi^{A}\right\rangle & =F_{2 a}^{A A} q_{2 a}+\frac{1}{2}\left[F_{11}^{A A} q_{1}^{2}+F_{2 a 2 a}^{A A} q_{2 a}^{2}+F_{2 b 2 b}^{A A} q_{2 b}^{2}+2 F_{12 a}^{A A} q_{1} q_{2 a}\right] \\
\left\langle\psi^{B}\left|V^{(2)}\right| \psi^{B}\right\rangle & =F_{2 a}^{B B} q_{2 a}+\frac{1}{2}\left[F_{11}^{B B} q_{1}^{2}+F_{2 a 2 a}^{B B} q_{2 a}^{2}+F_{2 b 2 b}^{B B} q_{2 b}^{2}+2 F_{12 a}^{B B} q_{1} q_{2 a}\right] \\
\left\langle\psi^{A}\left|V^{(2)}\right| \psi^{B}\right\rangle & =F_{2 b}^{A B} q_{2 b}+F_{2 a 2 b}^{A B} q_{2 a} q_{2 b}+F_{2 b 1}^{A B} q_{2 b} q_{1}
\end{aligned}
$$

where the F's represent the derivatives of electronic potential as defined by Equation 10 .

In spectroscopic analyses, the linear, quadratic, and bilinear JT coupling constants are typically cast as in terms of constants $D_{2}, K_{2}$, and $B_{12}$ respectively. $D_{2}$ and $K_{2}$ are unitless ratios given[2]

$$
\begin{aligned}
D_{2}=\frac{\epsilon_{2}}{\omega_{2}} & =2\left[\frac{F_{2 a}^{A B}}{\left(F_{2 a 2 a}^{A A}+F_{2 a 2 a}^{B B}\right)}\right]^{2} \\
K_{2} & =\frac{\left(F_{2 a 2 a}^{A A}-F_{2 a 2 a}^{B B}\right)}{F_{2 a 2 a}^{A A}+F_{2 a 2 a}^{B B}}
\end{aligned}
$$

where $\epsilon_{2}$ is the JT stabilization energy in the linear JT model.

The harmonic frequencies are given by

$$
\begin{aligned}
& \omega_{1}=F_{11}^{A A} \\
& \omega_{2}=\frac{F_{2 a 2 a}^{A A}+F_{2 a 2 a}^{B B}}{2}=\frac{F_{2 b 2 b}^{A A}+F_{2 b 2 b}^{B B}}{2},
\end{aligned}
$$

The bilinear coupling coefficient $B_{12}$ can be taken to be simply

$$
B_{12} \equiv F_{12 a}^{A B} .
$$

Since $\mathrm{Li}_{3}$ is "simple" (it has only one degenerate mode), a corresponding cross-quadratic coupling coefficient, spectroscopically typically denoted,[55] $\mathrm{C}_{i j}$, is not present. However for 
generality we note that $C_{i j} \equiv F_{i j}^{A B}$ where $i$ and $j$ are different JT active degenerate modes.

\subsubsection{Fitting Procedure}

The vibronic eigenvalues of the quadratic JT Hamiltonian are fit to experimental (or, in the present case, "artificial" calculated) data by varying the parameters of $\hat{\mathcal{H}}^{(2)}$. To accomplish this, we have developed in-house software, SOCJT2, designed to generate the quadratic JT Hamiltonian, compute its eigenvalues, and fit the level positions by varying the quadratic parameters using a Levenberg-Marquardt non-linear least squares fit (LSF) algorithm.[81, 82] SOCJT2 uses a direct product basis of electronic and nuclear basis functions to calculate the vibronic eigenvalues and provides results equivalent to those from the approach of Köppel, et al.[80]

The vibronic eigenvalues, $E_{i}^{(2)}(\mathbf{z})$, of $\mathcal{H}_{J T}^{(2)}$ calculated in SOCJT2 depend on the quadratic parameters defined above that are generically denoted by z. SOCJT2 then assigns the calculated eigenvalues to available vibronic energies, $E_{i}^{\exp }$, using the symmetry quantum number. For example, the fifth $e^{\prime}$ level from SOCJT2's calculation is assigned to the fifth "experimental" $e^{\prime}$ level. Then the root means squared (RMS) error defined as

$$
\mathrm{RMS}=\min _{\mathbf{z}} \sqrt{\frac{1}{M} \sum_{i=1}^{M}\left[E_{i}^{(2)}(\mathbf{z})-E_{i}^{\exp }\right]^{2}}
$$

is minimized with respect to the quadratic parameters using the Levenberg-Marquardt algorithm $[81,82]$ to obtain the final parameter values $(\mathbf{z})$. Here, $M$ denotes the number of data points being fit.

It may seem appealing to retain cubic, quartic, and higher terms in the Taylor series expansion of the potential. This is not practical for the purpose of fitting experimental results since the number of parameters increases very rapidly with the order of the expansion. In observed spectra, the quantity of data is typically less than 25 vibronic levels. It would not be useful to fit a large number of parameters to this limited data set, and for this reason, experimental analyses usually are based on the quadratic JT Hamiltonian or a subset thereof. 
In cases where the Taylor expansion for the true potential converges rapidly, the quadratic expansion may be sufficient. However, this will not always be the case, and a potential may contain significant cubic, quartic, and perhaps even higher order terms. In that case, the fit quadratic parameters would be potentially suspect due to trancation error, and one - at least upon initial reflection-might expect the fit to be poor.

\subsection{Theoretical Methodology}

\subsubsection{Quantum Chemical Calculations}

The two adiabatic potential energy surfaces of the lowest ${ }^{2} E^{\prime}$ electronic state of $\mathrm{Li}_{3}$ were calculated by the equation of motion coupled-cluster method known as EOMEA-CCSDT $[83,84]$. In these calculations, a similiarity transformed (effective) electronic Hamiltonian is constructed for the (closed-shell) $\mathrm{Li}_{3}$ cation, and subsequently diagonalized in the space of nine-electron determinants that correspond to the neutral species. The parameters used in the construction of the effective Hamiltonian are those from the coupled-cluster singles, doubles and triples (CCSDT) wavefunction [85] for the cation, and the diagonalization space comprises all determinants that correspond to addition of one electron to the virtual cation molecular orbitals, as well as those related to them by promotion of one and two electrons from occupied (in the cation) to unoccupied orbitals. The treatment of valence electron correlation in $\mathrm{Li}_{3}$ by this level of theory is exact, and the basis set used in the calculations was the augmented double zeta atomic natural orbital basis of Roos [86].

The adiabatic potentials of the $a_{1}$ and $b_{2}$ components of the ${ }^{2} E^{\prime}$ state were evaluated on a grid of points restricted to the $C_{2 v}$ subspace of nuclear displacements surrounding the minimum energy $D_{3 h}$ conical intersection $\left(r_{L i L i}=2.909 \AA\right)$, and then fit to polynomials. Care was taken in choosing both the number of displaced points and degree of polynomial in the fit so that the resulting force constants (in reduced normal coordinates) are accurate to $c a$. $0.1 \mathrm{~cm}^{-1}$. This information is sufficient to construct the JT Hamiltonian described in the next subsection. All quantum chemistry calculations were performed with CFOUR [87]. 


\subsubsection{Explicit Vibronic Hamiltonian}

For the $a b$ initio treatment we use the quartic Hamiltonian,

$$
\mathcal{H}_{J T}^{(4)}=T_{N}+V_{J T}^{(2)}+V_{J T}^{(4)}
$$

where

$$
\left\langle\psi^{i}\left|\hat{V}^{(4)}\right| \psi^{i^{\prime}}\right\rangle=\frac{1}{6} \sum_{k l m} F_{k l m}^{i i^{\prime}} q_{k} q_{l} q_{m}+\frac{1}{24} \sum_{k l m n} F_{k l m n}^{i i^{\prime}} q_{k} q_{l} q_{m} q_{n}
$$

In order to determine the diabatic electronic basis force constants $F_{k \ldots l}^{i i^{\prime}}$ through quartic, it is (with the exception of those quartic constants involving only the degenerate mode, see below) sufficient to determine the force fields of the two adiabatic electronic states that involve only the modes $q_{1}$ and $q_{a}$, as the diabatic and adiabatic surfaces are coincident at $C_{2 v}$ geometries. Relatively simple symmetry relations can then be used to obtain the full quartic force field in this limited potential in the diabatic basis $\left(F_{k \ldots l}^{i i^{\prime}}\right)$ from that in the adiabatic basis $\left(f_{k \ldots l}^{i}\right)$, as documented in the Table 1. Accordingly we can rewrite the matrix elements $\left\langle\psi^{i}\left|V^{(2)}\right| \psi^{i^{\prime}}\right\rangle$ in Eq. 10 as

$$
\begin{aligned}
\left\langle\psi^{A}\left|V^{(2)}\right| \psi^{A}\right\rangle & =f_{2 a}^{A} q_{2 a}+\frac{1}{2}\left[f_{11}^{A} q_{1}^{2}+\left(f_{2 a 2 a}^{A} q_{2 a}^{2}+f_{2 a 2 a}^{B}\right) q_{2 a}^{2}\right]+f_{12 a}^{A} q_{1} q_{2 a} \\
\left\langle\psi^{B}\left|V^{(2)}\right| \psi^{B}\right\rangle & =-f_{2 a}^{A} q_{2 a}+\frac{1}{2}\left[f_{11}^{A} q_{1}^{2}+\left(f_{2 a 2 a}^{B}+f_{2 a 2 a}^{A}\right) q_{2 a}^{2}\right]-f_{12 a}^{A} q_{1} q_{2 a} \\
\left\langle\psi^{A}\left|V^{(2)}\right| \psi^{B}\right\rangle & =f_{2 a}^{A} q_{2 b}+\frac{1}{2}\left(f_{2 a 2 a}^{B}-f_{2 a 2 a}^{A}\right) q_{2 a} q_{2 b}+f_{12 a}^{A} q_{1} q_{2 b}
\end{aligned}
$$

In the absence of quartic JT coupling, the constants $F_{2 a 2 a 2 a 2 a}^{i i^{\prime}}, F_{2 a 2 a 2 a 2 b}^{i i^{\prime}}, F_{2 a 2 a 2 b 2 b}^{i i^{\prime}}, F_{2 a 2 b 2 b 2 b}^{i i^{\prime}}$, and $F_{2 b 2 b 2 b 2 b}^{i i^{\prime}}$ obey the condition $i=i^{\prime}$ together with

$$
F_{2 a 2 a 2 a 2 a}^{A A}=F_{2 a 2 a 2 a 2 a}^{B B}=F_{2 b 2 b 2 b 2 b}^{A A}=F_{2 b 2 b 2 b 2 b}^{B B}=3 F_{2 a 2 a 2 b 2 b}^{A A}=3 F_{2 a 2 a 2 b 2 b}^{B B}=f_{2 a 2 a 2 a 2 a}^{A A} .
$$


This approximation was used in the construction of the JT Hamiltonian used in this work; the magnitude of diagonal quartic JT coupling is likely to be negligible, as evidenced by the fact that the adiabatic force constants $f_{2 a 2 a 2 a 2 a}^{A}$ and $f_{2 a 2 a 2 a 2 a}^{B}$ (which coincide in the absence of coupling) were small and similar to one another $\left(5.5\right.$ and $6.6 \mathrm{~cm}^{-1}$, respectively). It should be emphasized here that use of the equations relating the $F$ and $f$ force constants presupposes that the quantum chemical method used provides a proper treatment of the degenerate states, and thereby automatically satisfies the usual symmetry properties governing the force constants associated with modes 1 and $2 a$ found in a JT system, such as $f_{2 a}^{A}=-f_{2 a}^{B}$. The EOMEA-CC method is such an approach.[3]

\subsection{Comparing Experimental and Theoretical Methodologies}

\subsubsection{Scaling Cubic and Quartic Derivative Terms}

As mentioned earlier, one important possible source of discrepancy between experimental and theoretical analyses may be that the quadratic potential, $\hat{V}^{(2)}$, used by the "experimental" analysis, is insufficient. A goal of this work is to analyze how the inclusion of higher order terms affects fitted values of the quadratic JT coupling parameters, as well as the quality of the fit itself. This can be quantified in a controlled environment by fitting an "artificial spectrum" obtained by calculating vibronic energies using a known, "true" potential, which we suppose to be the quartic expansion, $\hat{V}^{(4)}$, from Equation (17).

Although we call these spectra "artificial," we still want our work to reflect meaningfully on the $\mathrm{Li}_{3}$ molecule. Hence, the ab initio calculation performed for $\mathrm{Li}_{3}$ (from which the force constants in $\hat{V}^{(2)}$ and $\hat{V}^{(4)}$ are calculated) was an accurate one. The procedure is to "turn on" the cubic and quartic terms by multiplying them by a scaling factor, $s$, between 0 and 1. To accomplish this, $\mathcal{H}_{J T}^{(4)}$ is parametrized by $s$ in the form

$$
\mathcal{H}_{J T}^{(4)}(s)=T_{N}+\mathcal{H}_{J T}^{(2)}+s \mathcal{H}_{J T}^{(4)}
$$

The $\mathcal{H}_{J T}(s)$ Hamiltonian can be diagonalized to yield vibronic energies $\left\{E_{i}^{(4)}(s)\right\}_{i}$ that 
depend on $s$. Instead of fitting experimental data as Equation (15) implies, we will instead fit $\left\{E_{i}^{(4)}(s)\right\}_{i}$ for various values of $s$ by minimizing

$$
\operatorname{RMS}(s)=\min _{z(s)} \sqrt{\frac{1}{M} \sum_{i}^{M}\left[E_{i}^{(2)}(\mathbf{z}(s))-E_{i}^{(4)}(s)\right]^{2}}
$$

with respect to quadratic parameters $\mathbf{z}(s)$ at each value of $s$. This will yield values for the second order parameters depending on $s: \omega_{k}(s), D_{d}(s), K_{d}(s), B_{t d}(s)$, and $C_{d d^{\prime}}(s)$. At $s=0$, the quadratic and quartic JT Hamiltonian are equivalent, so the second order parameters will coincide, and the quadratic fit will be exact. As $s$ increases, the change in quadratic parameter values reveals the desired knowledge: how they are affected by inclusion of cubic and quartic terms. This procedure mimics a purely experimental investigation of the questions at hand; the theoretical calculations serve only to supply (the possibly voluminous) set of level observations and assignments, thereby removing any potential ambiguities associated with incomplete data or incorrect assignments.

\subsubsection{Variation of Data Set Size}

There is another aspect of the analysis that involves the number of levels, $M$, included in the fit. As mentioned in Section 3.1.2, this number is usually less than 25 when analyzing experimental spectra. However, because we are fitting artificial spectra, we can fit as many levels as we choose, and then observe how the quadratic fit parameters (and the quality of the fit) change as the number of levels increase. If the quadratic potential is fundamentally inadequate, one would expect the RMS error of the fit to grow, perhaps frightfully, as the number of levels in the fit increases.

When $\hat{\mathcal{H}}_{J T}^{(4)}(s)$ is diagonalized, a set of vibronic energies are obtained, $E_{i}^{(4)}(s)$, that may be ordered from lowest to highest. To change the number of levels in the fit, we take subsets $\mathcal{E}$ of the set of these energies, each subset of different size.

$$
\mathcal{E}(s, \epsilon)=\left\{E_{k}^{(4)}(s) \text { such that } E_{k}^{(4)}(s)-E_{1}^{(4)}(s)<\epsilon\right\}
$$


for some energy cutoff $\epsilon$, and all vibronic energy levels less than $\epsilon$ above the lowest level will be fit. We can change this cutoff and observe the resulting change in parameter values and quality of the fit. For different values of $s$ and $\epsilon$, we will minimize

$$
\operatorname{RMS}(s, \epsilon)=\min _{\mathbf{z}(s, \epsilon)} \sqrt{\frac{1}{|\mathcal{E}(s, \epsilon)|} \sum_{i=1}^{|\mathcal{E}(s, \epsilon)|}\left[E_{i}^{(2)}(\mathbf{z}(s, \epsilon))-E_{i}^{(4)}(s)\right]^{2}}
$$

with respect to the quadratic parameters.

\section{Results}

As discussed in Section 1, $\mathrm{Li}_{3}$ exhibits the ubiquitous $E \otimes e$ JT effect along the degenerate vibrational mode, $\nu_{2}$ in its $\tilde{X}^{2} E^{\prime}$ state. Extensive theoretical[72, 73, 74, 75, 77, 76, 79, 88] and experimental[$[78,77,79]$ analyses have involved the JT effect in the $\tilde{X}^{2} E^{\prime}$ state. $\mathrm{Li}_{3}$ is particularly interesting because theory and experiment disagree on the magnitude of the

JT distortion in the $\tilde{X}^{2} E^{\prime}$ state. A potential culprit is the issue of fitting an inherently higher order potential expansion using only quadratic JT parameters. We have conducted a three-fold analysis by (i) reanalyzing previous experimental data from Dugourd, et al.[78] in light of newer experimental insight,[79] (ii) performing our own computational analysis, and (iii) using the methodology outlined in Section 3.3 to compare results from JT models using different expansion orders.

\subsection{Fitting Experimental Data}

Dugourd, et al.[78] have presented the most complete vibronic spectrum of the $\tilde{X}^{2} E^{\prime}$ state.[78] They fit the spectrum using a Hamiltonian equivalent to the quadratic JT Hamiltonian presented in Section 3.1 and we have reproduced the results by fitting the data with our quadratic JT Hamiltonian and obtaining the same values for the JT coupling parameters, which are given in Table 2

Work by Meyer and co-workers[79] has reported the first three lower lying vibronic levels in the $\tilde{X}^{2} E^{\prime}$ state. The level originally reported[78] at $66 \mathrm{~cm}^{-1}$ was not seen in this work. 
Our computational analysis, discussed in Section 4.2, also does not find a level that could reasonably be associated with such a state. In light of these developments, we removed the level at $66 \mathrm{~cm}^{-1}$ and refit the data using the methodology described in Section 3.1. We will refer to this fit as Refit I. The parameters from this fit are also listed in Table 2.

Furthermore, in our computational analysis, the level earlier reported[78] at $205 \mathrm{~cm}^{-1}$ also seems to be absent in the "artificial" spectrum. Considering that both this and the 66 $\mathrm{cm}^{-1}$ level may both be artifacts in earlier data, we performed another fit of the experimental data, this time removing both the level at $66 \mathrm{~cm}^{-1}$ and $205 \mathrm{~cm}^{-1}$. We will refer to this set as Refit II. The parameter values obtained from this fit are also listed in Table 2.

As can be seen from Table 2, the three sets of fit parameter values differ significantly. Also listed are the JTSE and pseudo-rotation barrier of each fit. The original experimental fit predicts a JTSE of $267 \mathrm{~cm}^{-1}$ and a barrier of $25.5 \mathrm{~cm}^{-1}$. The JTSE and barrier are lower than both Refit I and Refit II. The barrier heights predicted by Refit I and Refit II are both substantially higher than originally predicted at $71.2 \mathrm{~cm}^{-1}$ and $112 \mathrm{~cm}^{-1}$ respectively, and the JTSE for Refit II is significantly higher than both early results and Refit I at 386 $\mathrm{cm}^{-1}$. Refits I and II clearly suggest that one or two bad data points can completely throw off the experimental determination of the potential parameters. However it is hard to spot a problem based upon the quality of the experimental fits alone; some help from reliable theory is necessary.

Figure 1 shows the PES of the fitted quadratic potential along $q_{2 a}$ for each of the three fit results. Besides the differences in JTSE and pseudo-rotation barrier, we see that the geometry of the global minimum and of the barrier [which differ as to whether the Li-Li-Li angle bisected by the $\mathrm{C}_{2}$ axis is acute or obtuse] has switched between the fit of Dugourd, et al. and Refit I/II. Also plotted on this figure is the ab initio PES to be discussed in Section 4.2. We see that Refit I and Refit II predict somewhat more similar minimum and pseudorotational barrier geometries to the $a b$ initio calculation. It is also seen that the JTSE of Refit II best matches the $a b$ initio calculation, but Refit I best matches the $a b$ initio barrier 
height.

The levels calculated from the quadratic JT Hamiltonian and their symmetry assignments are listed in Table 3 alongside the experimental levels. It should be noted that no rotationally resolved spectra of the vibronic levels in the $\tilde{X}^{2} E^{\prime}$ state have been produced, and it is currently not possible to discern the symmetry of the vibronic levels by experimental means alone. The RMS errors of the fits are also listed in this table. The largest RMS error is Refit II with $6.52 \mathrm{~cm}^{-1}$. Given the resolution of the experimental vibronic spectrum, all of these fits could be considered good, although the fit parameters from the three approaches are strikingly different.

\subsection{Theoretical Analysis}

The most recent theoretical analysis was done by Ghassemi et al.[76] We have conducted our own theoretical analysis of the $\tilde{X}^{2} E^{\prime}$ state. The PES for the $\tilde{X}^{2} E^{\prime}$ state was computed at the EOMEA-CCSDT level of theory, and a JTSE of $505 \mathrm{~cm}^{-1}$ and pseudo-rotation barrier of $77.5 \mathrm{~cm}^{-1}$ were obtained. These are in essentially perfect agreement with the MRCI calculation which predicted a JTSE and barrier of 504 and $77 \mathrm{~cm}^{-1}$ respectively.[76] Significantly, however, they stand in contrast to the results obtained by the quadratic JT analyses of the experimental data, as summarized in Table 2.

The derivatives determined from $a b$ initio calculations are listed in Table 4. The quartic JT Hamiltonian based on these values was used to calculate a vibronic spectrum and the resulting spectrum is listed in Table 3 alongside the spectra obtained from the fits described in Section 4.1. The spectrum calculated from the quartic JT Hamiltonian matches the experimental spectrum very well, with the aforementioned absence of levels near 66 and $205 \mathrm{~cm}^{-1}$. As noted earlier, the $66 \mathrm{~cm}^{-1}$ level was also not seen by Kramer et al.[79] and

we suspect the level at $205 \mathrm{~cm}^{-1}$ is a similar anomaly. The symmetry assignments of the levels disagree significantly compared to the symmetry assignments made by Dugourd, et al.[78], but we now have an experimental and theoretical analysis that both reproduce the experiment level positions well even though they disagree substantially on the strength of 
JT coupling.

\subsection{Scaled Fits of $\mathrm{Li}_{3}$}

One potential reason for the behavior noted may be that the experimental fit was done with a quadratic JT Hamiltonian, whereas the ab initio vibronic spectrum was calculated from a quartic JT Hamiltonian. To test this, the method described in Section 3.3 was applied using the quartic terms in Table 4. The scaling parameter $s$ was incremented in steps of 0.01 from 0 to 1 and three energy cutoffs, $\epsilon$, are used: $400 \mathrm{~cm}^{-1}$ (the experimental data range), $800 \mathrm{~cm}^{-1}$, and $1200 \mathrm{~cm}^{-1}$. These energy cutoffs approximately correspond to fitting 15,30 , and 70 levels respectively. The parameters determined by these fits are pictured in Figure 2 as a function of $s$.

\section{Discussion}

\subsection{Convergence of the Ab Initio Potential}

There is reason to believe that the ab initio potential is closer to the true potential than that extracted from the analysis.[78] First, the fact that both MRCI and EOMEACCSDT (which are two quite different approaches) give almost exactly the same results lends credibility to the $a b$ initio potentials.[76] Moreover, if we believe that the level at 66 $\mathrm{cm}^{-1}$ and perhaps the level at $205 \mathrm{~cm}^{-1}$ are artifacts, then the $a b$ initio potential predicts JT coupling that is in better agreement with the Refit I and Refit II results.

It is a difficult question to determine whether the truncated potentials are sufficient to approximate the true potential of the $\tilde{X}^{2} E^{\prime}$ state. However, we can at least test for convergence of the analytical quadratic potential to the ab initio quartic potential for the $\tilde{X}^{2} E^{\prime}$ state. To do so, we can form two different potentials. One is the quartic potential that we have discussed thus far, with the parameters in Table 4 . The second potential is the same potential, but with all cubic and quartic derivatives in Table 4 set to zero. The JTSE and pseudo-rotation barrier are predicted by these two potentials. The values of the JTSE and barrier predicted by $\hat{V}^{(4)}$ of 507 and $78 \mathrm{~cm}^{-1}$ are in very close agreement with the exact 
values from the $a b$ initio surface. The same cannot be said for $\hat{V}^{(2)}$ which yields values of 480 and $45 \mathrm{~cm}^{-1}$ respectively, as shown by the $\mathrm{s}=0$ values in Fig. $2 \mathrm{~g}$ and $2 \mathrm{~h}$. This tells us that the quartic expansion for the potential is well converged in the Franck-Condon region, vastly superior to the corresponding quadratic truncation. Moreover, the vibronic spectrum using the JT2 potential can be calculated and compared to the vibronic spectrum using JT4. The results are shown in Table 5. The vibronic energies are significantly different, another indication that the quadratic expansion is inadequate.

If the $a b$ initio potential is representative of the true potential, the need for a quartic potential is perhaps surprising given the quality of the fits to the experimental data using the quadratic JT Hamiltonian. The fit by Dugourd, et al. provides levels with an RMS of $4.45 \mathrm{~cm}^{-1}$, and even our own refits have an RMS error of only 4.62 and $6.52 \mathrm{~cm}^{-1}$. These errors are small and to many spectroscopists, would be considered characteristic of a good fit for a spectrum of this quality and resolution. However, the discussion above lends credibility to the ab initio result, which disagrees significantly with every fit. Despite the fact that the experimental fits may use a potential that is fundamentally incapable of reproducing the true spectrum, the quality of the fits as measured by the RMS is quite acceptable. Clearly, $\mathrm{Li}_{3}$ serves as interesting test case for the scaled fits. We will discuss the results of these fits in the following sections.

\subsection{Effect of Cubic and Quartic Terms}

\subsubsection{Parameter Trends}

Figure 2 contains the plots of all fitted parameters and their dependence on $s$. These plots detail how the parameters of the quartic potential are affected by varying magnitudes of cubic and quartic terms. Also plotted are the potential energy surfaces of the results from fits of $s=0, s=0.5$, and $s=1$ compared with each other and the ab initio potential energy surface in Figure 3.

The individual panels of Figure 2 deserve some discussion. Figure $2 \mathrm{a}$ is the plot of $\omega_{1}$. Although $\nu_{1}$ is not a JT active mode, $\omega_{1}$ is affected at the quadratic (and higher) order 
coupling between $\nu_{1}$ and $\nu_{2}$, a JT active mode. The bilinear coupling term $B_{12}$ increases approximately linearly, as seen in Figure 2e. In Figure 2b, $\omega_{2}$ is plotted and the largest deviation from its initial value is only about $10 \% . D_{2}$ is plotted in Figure 2c and it decreases quickly at higher $s$ values. This results in a decreased JTSE as seen in Figure $2 \mathrm{~g}$.

$K_{2}$ switches sign as $s$ increases, as shown in Figure 2d. Moreover, at $s=1$ for the cutoff of $1200 \mathrm{~cm}^{-1}$, the value of $K_{2}$ is -0.1343 , which differs in sign and (by nearly a factor of 3 ) magnitude from the value of 0.04857 , calculated from the second derivative of the $a b$ initio potential. This has a profound effect on the PES by switching the upper and lower diabatic PES and will be further discussed in Section 5.2.2. The JTSE of the ab initio potential is 505 $\mathrm{cm}^{-1}$ and this value is underestimated for all values of $s$ for each cutoff as shown in Figure $2 \mathrm{~g}$. On the other hand, the pseudo-rotation barrier for the ab initio potential is ultimately reproduced well for all cutoffs.

Graphs of the PES as $s$ increases are shown in Figure 3. The PES along the $q_{2 a}$ coordinate is plotted for the $s=0.00, s=0.50$, and $s=1.00$ fits, along with the ab initio PES. As $s$ increases, we see that the geometry of the minimum and the barrier switch, as mentioned above. We also see that the JTSE of the fitted potential ultimately decreases bringing the value well above the $a b$ initio value. However, the barrier of the $s=1.00$ fit matches the $a b$ initio barrier well and it seems that the lower JTSE allows for the curvature of the $s=1.00$ fit to match the curvature of the $a b$ initio PES surprisingly well.

\subsubsection{Switching of Diabatic Surfaces}

The most interesting parameter variation observed is that for $K_{2}$. The introduction of cubic and quartic terms causes a crossing of levels with $a_{1}^{\prime}$ and $a_{2}^{\prime}$ vibronic symmetry, as shown in Figure 4. This indicates a switching of the lower and upper components of the $\tilde{X}^{2} E^{\prime}$ state. The crossing also switches the qualitative geometries (acute v. obtuse) of the pseudo-rotation barrier and minimum on the lower adiabatic surface.

This effect can only be captured in the quadratic JT Hamiltonian by a change in the sign of $K_{2}$, which is exactly what we observe in Figure 2d. We note that the cusp in the JTSE 
and pseudo-rotational barrier (Figures $2 \mathrm{~g}$ and $2 \mathrm{~h}$ ) are a result of this surface switching.

In this case, the JTSE was greatly underestimated by the quadratic fit. However, the pseudo-rotation barrier ultimately agrees fairly well with the barrier of the $a b$ initio potential. One takeaway from this analysis is that a fit using an inadequate potential expansion may still be able to capture some characteristics of the JT coupling effectively, but still improperly label the lower and upper diabatic components of the degenerate electronic state and consequently err in associating symmetries to levels.

\subsubsection{RMS of Fits}

Perhaps the most important (and surprising) observation is found in the RMS error of the fits as a function of $s$ and $\epsilon$ which is shown in Figure $2 \mathrm{f}$. We emphasize that the quadratic potential used in fitting the experimental data is flawed since the true potential has important cubic and quartic terms. One of the questions posed in the introduction was how one could know when the model potential used in the fits was insufficient. The most obvious metric is the RMS fit error, which is widely checked as an indicator of fit quality in analyses. Following this mindset, we would expect $\operatorname{RMS}(s=1, \epsilon)$ to be quite high, knowing that the model potential is insufficient. However, the RMS of these fits as $s$ is increased does not increase greatly. Ultimately, $\operatorname{RMS}\left(s=1, \epsilon=400 \mathrm{~cm}^{-1}\right)=5.4 \mathrm{~cm}^{-1}, \operatorname{RMS}(s=1, \epsilon=800$ $\left.\mathrm{cm}^{-1}\right)=5.9 \mathrm{~cm}^{-1}$, and $\operatorname{RMS}\left(s=1, \epsilon=1200 \mathrm{~cm}^{-1}\right)=6.4 \mathrm{~cm}^{-1}$. Fits exhibiting these RMS values would be considered fairly good fits for many experiments, especially given that it is often impossible to determine vibronic origin positions to better than a few $\mathrm{cm}-1$.

It may not come as a surprise that the RMS error monotonically increases with $\epsilon$. Since fewer levels are being fit at lower $\epsilon$, the relatively large number of parameters may adapt to match these levels without having much physical significance. If more levels are added to the fit, the fitting Hamiltonian will become gradually unable to (deceptively) match each

level and its shortcomings will be exposed by a larger RMS. While the RMS values do increase with $\epsilon$ at $s=1$, it is only a $10 \%$ increase after adding nearly 5 times more levels to the fit. Moreover, while the parameters may change to different values, the general trend 
of parameters with $s$ is consistent throughout different truncations of the number of levels fit. This indicates that the low RMS is not a result of dramatic changes in the quadratic potential. The quadratic potential simply does a surprisingly good job of emulating the vibronic structure of the quartic potential, although the constants comprised by the best fit potential agree quite poorly with the "exact" constants. A critical point to take away from this is that here the RMS is not a sound objective measure of the sufficiency of the model potential. Thus, and as a warning to all experimental fits, of JT systems, a low RMS error does not necessarily mean that the potential is accurately represented; discrepancies with computed potentials should not always be attributed to "bad" calculations. Perhaps a better indicator of the reliability of inferred potential parameters from a quadratic fit is to see to what degree a calculated quadratic JT model - parametrized at the high symmetry point - is able to account for potential energy surface "benchmarks" like the JT stabilization energy, adiabatic potential surface minima and saddle point geometries, and pseudorotation barrier height. This can be done by means of comparison between the values obtained from the model to those obtained by direct calculation. If such disagreement is significant, it is reasonable to assume that the experimentally inferred parameters from the same model applied to the experimental level positions are similarly unreliable, irrespective of the quality of the fit to the data.

A possible explanation for the behavior noted above is based on the fact that the parameters of the quadratic Hamiltonian obtained from fitting the data cannot be interpreted at face value. That is, the effects of higher than quadratic terms in the physical Hamitonian are implicitly incorporated into the parameters of the truncated model. From the perspective of perturbation theory, some of the higher-order contributions can be "folded into" the parameters of quadratic model; it seems - although based on only the present example - that such a "redressed" truncated Hamiltonian may be able provide a good representation of the low-lying energy level structure. Further theoretical analysis of this issue is clearly in order, and an appropriate topic for future investigation. 


\subsubsection{Multiple Minima to the Least Squares Fit}

A point of lingering concern to address this is the apparent jaggedness and "edges" seen in the plots in Figure 2 where one reasonably expects smooth curves. The reason for this result is the hopping between different minima in the least squares fit. In a least squares fit, the RMS error is a function of the parameters and is minimized in the fit. There are multiple minima for the RMS error, corresponding to different sets of parameter values. As $s$ changes, the state energies change and thus, so does the RMS minimum and value of parameters at the minima. As $s$ increases, there is a chance that some of the local minima switch and the minimum which is the absolute minimum changes. When this occurs, the least squares fit converges to a different minimum and this appears as a jagged point or discontinuous jump in the parameter plots with respect to $s$.

This is highlighted in Figure 5. The plots of $\omega_{2}$ and RMS with respect to $s$ are plotted alongside each other for the cutoff of $1200 \mathrm{~cm}^{-1}$. Whenever there is a discontinuity or spike in the plot of $\omega_{2}$, there is a corresponding jagged feature in the RMS plot, indicating that the fit converged to a different minimum with a lower RMS. This observation is a testament to the difficulty of fitting experimental data. If there are multiple minima in the least squares fit, the fit may jump between them. This leads to dramatic changes in parameter values, despite small changes in RMS. Therefore, another reason becomes clear that the parameter values obtained through fits may be somewhat unstable and potentially rather unreliable.

\section{Conclusion}

A systematic study has been performed to quantify the effects of higher-order JT coupling terms on fits of both experimental and artificial vibronic spectra using a second-order JT Hamiltonian. We test the ability of a quadratic JT Hamiltonian to fit vibronic levels of the $\tilde{X}^{2} E^{\prime}$ state of $\mathrm{Li}_{3}$ as determined from experiment and from a calculated quartic potential. In

the latter case, the magnitude of the higher order terms can be varied from zero to a value of unity which recaptures the full calculated potential. This potential, which appears to a 
good approximation to the exact result in the relevant regions of the potential, accurately predicts the experimentally observed vibronic structure, with the omission of two reported transitions, which may be experimental artifacts. This illustrates how the JT parameters originally reported for $\mathrm{Li}_{3}$ can be improved by additional experimental results and reliable theory.

Graphical representations are presented showing the variation in the value of the fit JT parameters as the magnitude of the higher order terms are varied from 0 to their proper values. As expected, the fit parameters as well as the JT stabilization energy and barrier to pseudorotation change significantly. Surprisingly, the change in the RMS deviation for the fit to the transition frequencies of the "artificial" spectrum does not increase nearly as dramatically as changes in the fit parameters and potential might suggest. Moreover, even a several fold increase in the number of transitions included in the fit only moderately increases the RMS. These observations are rationalized by considering how the effect of the higher order potential terms may be diminished for the eigenvalues of the vibronic Hamiltonian, perhaps due to a selectivity imposed by the matrix elements of the vibrational coordinates.

These results have important practical impacts for understanding the relationship between experimental spectral fits and calculated potentials. It can now clearly be argued that a quadratic fit of reasonably high quality, i.e. low RMS, is not sufficient to conclude that the potential is well described by the resulting parameters. To compare calculated potentials with experiment requires a more stringent test, e.g. a good, non-adjusted prediction of the observed transition frequencies directly from the calculated potential.

\section{Acknowledgments}

An Undergraduate Research Scholarship from the OSU College of Arts and Sciences (HT) and the Camille and Henry Dreyfus Foundation Senior Scientist Mentor Program (TAM) are greatly acknowledged. JFS acknowledges support of the U. S. Department of Energy, Basic Energy Sciences (Grant DE-FG02-07ER15684). 
[1] H. A. Jahn and E. Teller. Stability of Polyatomic Molecules in Degenerate Electronic States. I. Orbital Degeneracy. Proc. Roy. Soc., 161:220, 1937.

[2] T. Barckholtz and T. A. Miller. Quantitative Insights About Molecules Exhibiting Jahn-Teller and Related Effects. Int'l. Rev. of Phys. Chem., 17:435, 1998.

[3] J. F. Stanton. Coupled-Cluster Theory, Pseudo-Jahn-Teller Effects and Conical Intersections. J. Chem. Phys., 115:10382, 2001.

[4] T. A. Miller and V. E. Bondybey. Molecular Ions: Spectroscopy, Structure, and Chemistry, chapter The Jahn-Teller Effect in Benzenoid Cations: Theory and Experiment, page 201. North-Holland, Amsterdam, 1983.

[5] T. J. Sears, T. A. Miller, and V. E. Bondybey. The Laser Induced Fluorescence Spectrum of the sym- Trichlorotrifluorobenzene Radical Cation in the Gas Phase. J. Am. Chem. Soc., 102:4864, 1980.

[6] T. J. Sears, T. A. Miller, and V. E. Bondybey. Jahn-Teller Distortions in $\mathrm{C}_{6} \mathrm{H}_{3} \mathrm{~F}_{3}^{+}$and $\mathrm{C}_{6} \mathrm{H}_{3} \mathrm{Cl}_{3}^{+}$. J. Chem. Phys., 72:6070, 1980.

[7] T. J. Sears, T. A. Miller, and V. E. Bondybey. The Jahn-Teller Effect in $\mathrm{C}_{6} \mathrm{~F}_{6}^{+}$. J. Chem. Phys., 74:3240, 1981.

[8] K. Raghavachari, R. C. Haddon, T. A. Miller, and V. E. Bondybey. Theoretical Study of Jahn-Teller Distortions in $\mathrm{C}_{6} \mathrm{H}_{6}^{+}$and $\mathrm{C}_{6} \mathrm{~F}_{6}^{+}$. J. Chem. Phys., 79:1387, 1983.

[9] D. Purins and H. F. Feeley. Jahn-Teller Effect in the Cyclopentadienyl Radical: An Example of Three Mode Coupling. J. Mol. Struct., 22:11, 1974.

[10] P. C. Engelking and W. C. Lineberger. Laser Photoelectron Spectrometry of $\mathrm{C}_{5} \mathrm{H}_{5}$ : A Determination of the Electron Affinity and Jahn-Teller Coupling in Cyclopentadienyl. J. Chem. Phys., 67:1412, 1977. 
[11] H. H. Nelson, L. Pasternack, and J. R. McDonald. Excitation and Emission Spectra of the ${ }^{2} \mathrm{~A}_{2}^{\prime \prime} \leftrightarrow{ }^{2} \mathrm{E}_{1}^{\prime \prime}$ System of the Gas-Phase Cyclopentadienyl Radical. Chem. Phys., 74:227, 1983.

[12] L. Yu, S. C. Foster, J. M. Williamson, M. C. Heaven, and T. A. Miller. Rotationally Resolved Electronic Spectrum of Jet-Cooled Cyclopentadienyl Radical. J. Phys. Chem., 92:4263, 1988.

[13] L. Yu, D. W. Cullin, J. M. Williamson, and T. A. Miller. High Resolution Laser Spectroscopy of Asymmetrically Deuterated Cyclopentadienyl Radicals: A Study of Vibronic Degeneracy Resolution and Jahn-Teller Distortion. J. Chem. Phys., 98:2682, 1993.

[14] A. D. Liehr. On the Stability of Cyclobutadiene, Cyclopentadienyl Radical, and the Benzene Plus One Ion: A Comparison of the Molecular Orbital and Valence Bond Predictions. Z. Phys. Chem., 9:338, 1956.

[15] L. C. Snyder. Jahn-Teller Distortions in Cyclobutadiene, Cyclopentadienyl Radical, and Benzene Positive and Negative Ions. J. Chem. Phys., 33:619, 1960.

[16] W. D. Hobey and A. D. McLachlan. Dynamical Jahn-Teller Effect in Hydrocarbon Radicals. J. Chem. Phys., 33:1695, 1960.

[17] R. Meyer, F. Graf, T.-K. Ha, and H. H. Gunthard. Jahn-Teller Effect in Cyclopentadienyl Radical: Delocalized Vibronic Valence Isomerisation. Chem. Phys. Lett., 66:65, 1979.

[18] E. P. F. Lee and T. G. Wright. A Study of the Lowest-Lying Triplet and Singlet States of the Cyclopentadienyl Cation $\left(\mathrm{C}-\mathrm{C}_{5} \mathrm{H}_{5}^{+}\right)$. Phys. Chem. Chem. Phys., 1:219, 1999.

[19] B. E. Applegate, A. J. Bezant, and T. A. Miller. The Jahn-Teller and Related Effects 
in the Cyclopentadienyl Radical, Part II: Vibrational Analysis of the $\tilde{A}^{2} \mathrm{~A}_{2}^{\prime \prime}-\tilde{X}^{2} \mathrm{E}_{1}^{\prime \prime}$ Electronic Transition. J. Chem. Phys., 114:4869, 2001.

[20] W. T. Borden and E. R. Davidson. Potential Surfaces for the Planar Cyclopentadienyl Radical and Cation. J. Am. Chem. Soc., 101:3771, 1979.

[21] B. E. Applegate, T. A. Miller, and T. A. Barckholtz. The Jahn-Teller and Related Effects in the Cyclopentadienyl Radical, Part I: The Ab Initio Calculation of Spectroscopically Observable Parameters. J. Chem. Phys., 114:4855, 2001.

[22] T. Ichino, S. W. Wren, K. M. Vogelhuber, A. J. Gianola, W. C. Lineberger, and J. F. Stanton. The Vibronic Level Structure of the Cyclopentadienyl Radical. J. Chem. Phys., 129:084310, 2008.

[23] P. G. Carrick, S. D. Brossard, and P. C. Engelking. Experimental Observation of the Spin-Orbit Splitting in Methoxy. J. Chem. Phys., 83:1995, 1985.

[24] S. D. Brossard, P. G. Carrick, E. L. Chappell, S. C. Hulegaard, and P. C. Engelking. The ${ }^{2} \mathrm{~A}_{1^{-}}{ }^{2}$ E Electronic Spectrum of Methoxy, $\mathrm{CH}_{3} \mathrm{O}$ : Vibrational Progressions, and SpinOrbit and Jahn-Teller Couplings. J. Chem. Phys., 84:2459, 1986.

[25] X. Liu, S. C. Foster, J. M. Williamson, L. Yu, and T. A. Miller. The Spin-Rotation Interactions in the Methoxy Radical. Mol. Phys., 69:357, 1990.

[26] X. Liu, L. Yu, and T. A. Miller. Jahn-Teller Induced Corrections to Rotational and Fine-Structure Parameters in Doubly Degenerate Electronic States. J. Mol. Spectrosc., 140:112, 1990.

[27] D. E. Powers, M. Pushkarsky, and T. A. Miller. Vibrational Mode and Frequency Dependence of the Photofragmentation of the Methoxy Radical. J. Chem. Phys., 106:6878, 1997. 
[28] S. C. Foster, P. Misra, T. Y. D. Lin, C. P. Damo, C. C. Carter, and T. A. Miller. The Free Jet-Cooled Laser-Induced Fluorescence Spectrum of Methoxy. Part I: Vibronic Analysis of the $\widetilde{A}$ and $\widetilde{X}$ States. J. Phys. Chem., 92:5914, 1988.

[29] G. Inoue, H. Akimoto, and M. Okuda. Laser-Induced Fluorescence Spectra of $\mathrm{CH}_{3} \mathrm{O}$. Chem. Phys. Lett., 63:213, 1979.

[30] Y.-Y. Lee, G.-H. Wann, and Y.-P. Lee. Vibronic Analysis of the $\widetilde{A} \rightarrow \widetilde{X}$ Laser-Induced Fluorescence of Jet-Cooled Methoxy $\left(\mathrm{CH}_{3} \mathrm{O}\right)$ Radical. J. Chem. Phys., 99:9465-9471, 1993.

[31] A. Geers, J. Kappert, and F. Temps. Stimulated Emission Pumping Spectroscopy of $\mathrm{CH}_{3} \mathrm{O}\left(\mathrm{X}^{2} \mathrm{E}, \nu_{6}\right)$ : New Observations on the Jahn-Teller Effect. J. Chem. Phys., 98:42974300, 1993.

[32] A. Geers, J. Kappert, F. Temps, and J. Wiebrecht. Rotation-Vibration State Resolved Unimolecular Dynamics of Highly Vibrationally Excited $\mathrm{CH}_{3} \mathrm{O}\left(\mathrm{X}{ }^{2} \mathrm{E}\right)$. I. Observed Stimulated Emission Pumping Spectra. J. Chem. Phys., 101:3618, 1994.

[33] A. G. J. Kappert, F. Temps, and J. Wiebrecht. Rotation-Vibration State Resolved Unimolecular Dynamics of Highly Vibrationally Excited $\mathrm{CH}_{3} \mathrm{O}\left(\mathrm{X}^{2} \mathrm{E}\right)$. II. Intramolecular Vibrational Dynamics of Excited "C-O Stretch" States. J. Chem. Phys., 101:3634, 1994.

[34] M. Iwasaki and K. Toryama. A Simple Model for Hydrogen Atom Reactions in Neopentane-Cyclohexane Mixtures Irradiated at 4.2K. J. Am. Chem. Soc., 100:1964$1965,1978$.

[35] M. J. Nee, A. Osterwalder, J. Zhou, and D. M. Neumark. Slow Electron Velocity-map Imaging Photoelectron Spectra of the Methoxide Anion. J. Chem. Phys., 125:014306/1014306/9, 2006. 
[36] T. M. Ramond, G. E. Davico, R. L. Schwartz, and W. C. Lineberger. Vibronic Structure of Alkoxy Radicals via Photoelectron Spectroscopy. J. Chem. Phys., 112:1158, 2000.

[37] C. L. Osborn, D. J. Leahy, E. M. Ross, and D. M. Neumark. Study of the Predissociation of $\mathrm{CH}_{3} \mathrm{O} \widetilde{A}^{2} \mathrm{~A}_{1}$ by Fast Beam Photofragment Translational Spectroscopy. Chem. Phys. Lett., 235:484, 1995.

[38] D. L. Osborn, D. J. Leahy, and D. M. Neumark. Photodissociation Spectroscopy and Dynamics of $\mathrm{CH}_{3} \mathrm{O}$ and $\mathrm{CD}_{3} \mathrm{O}$. J. Chem. Phys. A, 101:6583, 1997.

[39] H. E. Radford and D. K. Russell. Spectroscopic Detection of Methoxy $\left(\mathrm{CH}_{3} \mathrm{O}\right)$. J. Chem. Phys., 66:2222, 1977.

[40] D. K. Russell and H. E. Radford. Analysis of the LMR Spectra of Methoxy, $\mathrm{CH}_{3} \mathrm{O} . J$. Chem. Phys., 72:2750, 1980.

[41] Y. Endo, S. Saito, and E. Hirota. The Microwave Spectrum of the Methoxy Radical $\mathrm{CH}_{3}$ O. J. Chem. Phys., 81:122, 1984.

[42] T. Momose, Y. Endo, E. Hirota, and T. Shida. The Submillimeter-Wave Spectrum of the ${ }^{13} \mathrm{CH}_{3} \mathrm{O}$ Radical. J. Chem. Phys., 88:5338, 1988.

[43] T. Momose, Y. Endo, E. Hirota, and T. Shida. Erratum: The Submillimeter-Wave Spectrum of the ${ }^{13} \mathrm{CH}_{3} \mathrm{O}$ Radical [J. Chem. Phys. 88, 5338 (1988)]. J. Chem. Phys., 90:4636, 1989.

[44] J. Liu, M.-W. Chen, D. Melnik, J. T. Yi, and T. A. Miller. The Spectroscopic Characterization of the Methoxy Radical (Part I): Rotationally Resolved $\tilde{A}^{2} \mathrm{~A}_{1}-\tilde{X}^{2} \mathrm{E}$ Electronic Spectra of $\mathrm{CH}_{3}$ O. J. Chem. Phys., 130:074302, 2009.

[45] J. Liu, M.-W. Chen, D. Melnik, T. A. Miller, Y. Endo, and E. Hirota. The Spectroscopic Characterization of the Methoxy Radical (Part II): Rotationally Resolved $\tilde{A}^{2} \mathrm{~A}_{1}-\tilde{X}^{2} \mathrm{E}$ 
Electronic and $\tilde{X}^{2} \mathrm{E}$ Microwave Spectra of the Perdeuteromethoxy Radical $\mathrm{CD}_{3} \mathrm{O} . J$. Chem. Phys., 130:074303, 2009.

[46] D. Melnik, J. Liu, M.-W. Chen, and T. A. Miller. The Spectroscopic Characterization of the Methoxy Radical (Part III): Rotationally Resolved $\tilde{A}^{2} \mathrm{~A}_{1}-\tilde{X}^{2} \mathrm{E}$ Electronic Spectra of $\mathrm{CH}_{2} \mathrm{DO}$ and $\mathrm{CHD}_{2} \mathrm{O}$. J. Chem. Phys., 135:094310, 2011.

[47] B. E. Applegate, M. B. Pushkarsky, and T. A. Miller. Spectral Analysis and Photofragmentation Dynamics of the Perdeuteromethoxy Radical. J. Phys. Chem., 103:1538, 1999.

[48] G. D. Bent, G. F. Adams, R. H. Bartram, G. D. Purvis, and R. J. Bartlett. Manybody Perturbation Theory Electronic Structure Calculations for the Methoxy Radical. I. Determination of JahnTeller Energy Surfaces, Spin-orbit Splitting, and Zeeman Effect. J. Chem. Phys., 76:8219-8232, 1994.

[49] A. V. Marenich and J. E. Boggs. A Variational Study of Nuclear Dynamics and Structural Flexibility of the $\mathrm{CH}_{2} \mathrm{OH}$ Radical. J. Chem. Phys., 119:3098-3105, 2003.

[50] A. V. Marenich and J. E. Boggs. A Model Spin-Vibronic Hamiltonian for Twofold Degenerate Electron Systems: A Variational ab initio Study of $\tilde{X}^{2} \mathrm{E} \mathrm{CH}_{3} \mathrm{O}$. J. Chem. Phys., 122:024308/1-024308/11, 2005.

[51] A. V. Marenich and J. E. Boggs. Equation-of-Motion Coupled-Cluster Study of JahnTeller effect in $\mathrm{X}^{2} \mathrm{E} \mathrm{CF}_{3} \mathrm{O}$ and $\mathrm{CF}_{3} \mathrm{~S}$. Inter. J. Quant. Chem., 106:2609-2616, 2006.

[52] A. V. Marenich and J. E. Boggs. The Molecular Structure, Spin-Vibronic Energy Levels, and Thermochemistry of $\mathrm{CH}_{3}$ O. J. Mol. Struct., 780-781:163-170, 2006.

[53] J. Nagesh and E. L. Sibert. Vibrational Dynamics Around the Conical Intersection: A Study of Methoxy Vibrations on the $\tilde{X}^{2} E$ Surface. Phys. Chem. Chem. Phys., 12:8250, 2010. 
[54] J. Nagesh and E. L. Sibert. Infrared Spectra at a Conical Intersection: Vibrations of Methoxy. J. Phys. Chem., pages 3846-3855, 2012.

[55] T. Codd, M.-W. Chen, M. Roudjane, J. F. Stanton, and T. A. Miller. Jet Cooled Cavity Ringdown Spectroscopy of the $\tilde{A}^{2} E^{\prime \prime} \leftarrow \tilde{X}^{2} A_{2}^{\prime}$ Transition of the $\mathrm{NO}_{3}$ Radical. J. Chem. Phys., 142:184305, 2015.

[56] R. P. Wayne, I. Barnes, P. Biggs, J. P. Burrows, C. E. Canosa-Mas, J. Hjorth, G. L. Bras, G. K. Moortgat, D. Perner, G. Poulet, G. Restelli, and H. Sidebottom. The Nitrate Radical: Physics, Chemistry, and the Atmosphere. Atmospheric Environment, 25:1-203, 1991.

[57] P. S. Monks. Gas-Phase Radical Chemistry in the Troposphere. Chem. Soc. Rev., $34: 376-395,2005$.

[58] E. J. Jones and O. R. Wulf. The Absorption Coefficient of Nitrogen Pentoxide in the Ultraviolet and the Visible Absorption Spectrum of $\mathrm{NO}_{3}$. J. Chem. Phys., 5:873, 1937.

[59] E. Hirota, K. Kawaguchi, T. Ishiwata, and I. Tanaka. Vibronic interactions in the $\mathrm{NO}_{3}$ radical. J. Chem. Phys., 95(2):771, July 1991.

[60] T. Ishiwata, I. Tanaka, K. Kawaguchi, and E. Hirota. Infrared Diode Laser Spectroscopy of the $\mathrm{NO}_{3} \nu_{3}$ Band. J. Chem. Phys., 82:2196, 1985.

[61] R. R. Friedl and S. P. Sander. Fourier Transform Infrared Spectroscopy of the $\mathrm{NO}_{3} \nu_{2}$ and $\nu_{3}$ Bands: Absolute Line Strength Measurements. J. Phys. Chem., 91:2721, 1987.

[62] K. Kawaguchi, E. Hirota, T. Ishiwata, and I. Tanaka. A Reinvestigation of the $\mathrm{NO}_{3}$ $1492 \mathrm{~cm}^{-1}$ Band. J. Chem. Phys., 93:951, 1990.

[63] K. Kawaguchi, T. Ishiwata, I. Tanaka, and E. Hirota. Fourier-Transform Infrared Spectroscopy of the $\mathrm{NO}_{3}$ Radical. Chem. Phys. Lett., 180:436, 1991. 
[64] K. Kawaguchi, T. Ishiwata, E. Hirota, and I. Tanaka. Infrared Spectroscopy of the $\mathrm{NO}_{3}$ Radical. Chem. Phys., 231:193, 1998.

[65] T. Ishiwata, Y. Nakano, K. Kawaguchi, E. Hirota, and I. Tanaka. Analyses of the Infrared Bands of ${ }^{15} \mathrm{NO}_{3}$ in the $1850-3150 \mathrm{~cm}^{-1}$ Region. J. Phys. Chem., 114:980-986, 2010.

[66] K. Kawaguchi, T. Ishiwata, I. Tanaka, and E. Hirota. Fourier-Transform Infrared Spectroscopy of the $\mathrm{NO}_{3}$ Radical. Chem. Phys. Lett., 180:436, 1991.

[67] J. F. Stanton. On the Vibronic Level Structure in the $\mathrm{NO}_{3}$ Radical: I. The Ground Electronic State. J. Chem. Phys., 126:134309, 2007.

[68] J. F. Stanton. On the Vibronic Level in the $\mathrm{NO}_{3}$ Radical: II. Adiabatic Calculation of the Infrared Spectrum. Mol. Phys., 107:1059, 2009.

[69] C. S. Simmons, T. Ichino, and J. F. Stanton. The $\nu_{3}$ Fundamental in $\mathrm{NO}_{3}$ Has Been Seen Near $1060 \mathrm{~cm}^{-1}$, Albeit Some Time Ago. J. Phys. Chem. Lett., 3:1946-1950, 2012.

[70] W. Eisfeld, O. Vieuxmair, and A. Viel. Full-dimensional Diabatic Potential Energy Surfaces Including Dissociation: The ${ }^{2} E^{\prime \prime}$ State of $\mathrm{NO}_{3}$. J. Chem. Phys., 140:224109, 2014.

[71] E. Hirota, T. Ishiwata, K. Kawaguchi, M. Fujitake, N. Ohashi, and I. Tanaka. NearInfrared Band of the Nitrate Radical $\mathrm{NO}_{3}$, Observed by Diode Laser Spectroscopy. $J$. Chem. Phys., 107:2829, 1997.

[72] W. H. Gerber and E. Schumacher. The Dynamic Jahn-Teller Effect in the Electronic Ground State of $\mathrm{Li}_{3}$. An ab initio Calculation of the BO Hypersurface and the Lowest Vibronic States of $\mathrm{Li}_{3}$. J. Chem. Phys., 69:1692, 1978.

[73] J. L. Martins, R. Car, and J. Buttet. Electronic Properties of Alkali Trimers. J. Chem. Phys., 78:5646, 1983. 
[74] T. C. Thompson, G. I. Jr., S. J. Lemon, D. G. Truhlar, and C. A. Mead. Consistent Analytic Representation of the Two Lowest Potential Energy Surfaces for $\mathrm{Li}_{3}, \mathrm{Na}_{3}$, and K. J. Chem. Phys., 82:5597, 1985.

[75] M. Ehara and K. Yamashita. Theoretical Studies of the Potential Energy Surface and Wavepacket Dynamics of the $\mathrm{Li}_{3}$ System. Theor. Chem. Acc., 102:226, 1999.

[76] E. N. Ghassemi, J. Larson, and A. Larson. A Diabatic Representation of the Two Lowest Electronic States of $\mathrm{Li}_{3}$. J. Chem. Phys., 120:2590, 2014.

[77] M. Keil, H.-G. Krämer, A. Kudell, M. A. Baig, J. Zhu, W. Demtröder, and W. Meyer. Rovibrational Structures of the Pseudorotating Lithium Trimer ${ }^{21} \mathrm{Li}_{3}$ : Rotationally Resolved Spectroscopy and ab initio Calculations of the $A^{2} E^{\prime \prime} \leftarrow X^{2} E^{\prime}$ System. J. Chem. Phys., 113:7414, 2000.

[78] P. Dugoard, J. Chevaleyre, M. Broyer, J. Wolf, and L. Wöste. Vibronic Structure of the $\mathrm{Li}_{3}$ Ground State. Chem. Phys. Lett., 175:555-560, 1990.

[79] H.-G. Krämer, M. Keil, C. B. Suarez, W. Demtröder, and W. Meyer. Vibrational Structures in the $A^{2} E^{\prime \prime} \leftarrow X^{2} E^{\prime}$ System of the Lithium Trimer: High-resolution Spectroscopy and ab initio Calculations. Chem. Phys. Lett., 299:212, 1999.

[80] H. Köppel, W. Domcke, and L. S. Cederbaum. Multimode Molecular Dynamics Beyond the Born-Oppenheimer Approximation. Adv. Chem. Phys., 57:59, 1984.

[81] K. Levenberg. A Method for the Solution of Certain Non-Linear Problems in Least Squares. Q. Appl. Math., 2:164-168, 1944.

[82] D. W. Marquardt, R. G. Bennett, and E. J. Burrell. Least Squares Analysis of Electron Paramagnetic Resonance Spectra. J. Mol. Spectrosc., 7:269-279, 1961. 
[83] M. Musial and R. J. Bartlett. Equation-of-Motion Coupled Cluster Method with Full Inclusion of Connected Triple Excitation for Electron-Attached States: EA-EOM-CCSDT. J. Chem. Phys., 119:1901, 2003.

[84] S. Hirata, M. Nooijen, and R. J. Bartlett. High-Order Determinantal Equation-ofMotion Coupled-Cluster Calculations for Ionized and Electron-Attached States. 328:459, 2000.

[85] J. Noga and R. J. Bartlett. The Full CCSDT Model for Molecular Electronic Structure. J. Chem. Phys., 86:7041, 1987.

[86] P.-O. Widmark, P. A. Malmqvist, and B. O. Roos. Density Matrix Averaged Atomic Natural Orbital (ANO) Basis Sets for Correlated Moelcular Wave Functions. I. First Row Atoms. Theor. Chim. Acta, 77:291, 1990.

[87] See www.cfour.de.

[88] W. Meyer, M. Keil, A. Kudell, M. A. Baig, J. Zhu, and W. Demtröder. The Hyperfine Structure in the Electronic $A^{2} E^{\prime \prime} \leftarrow X^{2} E^{\prime}$ System of the Pseudorotating Lithium Trimer. J. Chem. Phys., 115:154304, 2001. 


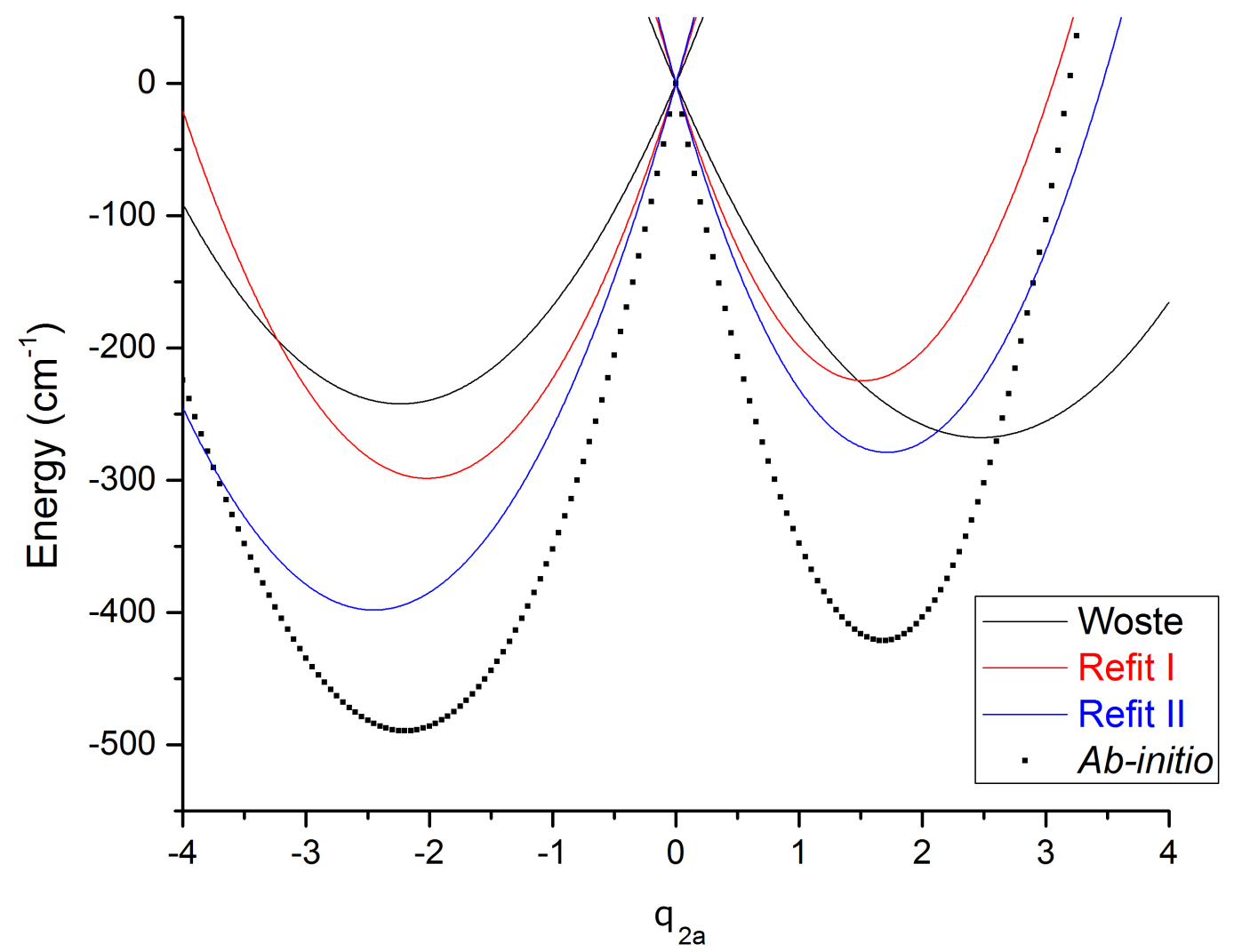

Figure 1: The PES of $\mathrm{Li}_{3}$ along one component of $\nu_{2}$ from (i) a fit by Dugourd, et al.,[78] (ii) a fit using the quadratic potential removing the level at $66 \mathrm{~cm}^{-1}$ denoted Refit I, (iii) a fit using the quadratic potential removing the levels at $66 \mathrm{~cm}^{-1}$ and at $205 \mathrm{~cm}^{-1}$ denoted Refit II, and the ab initio calculation. 


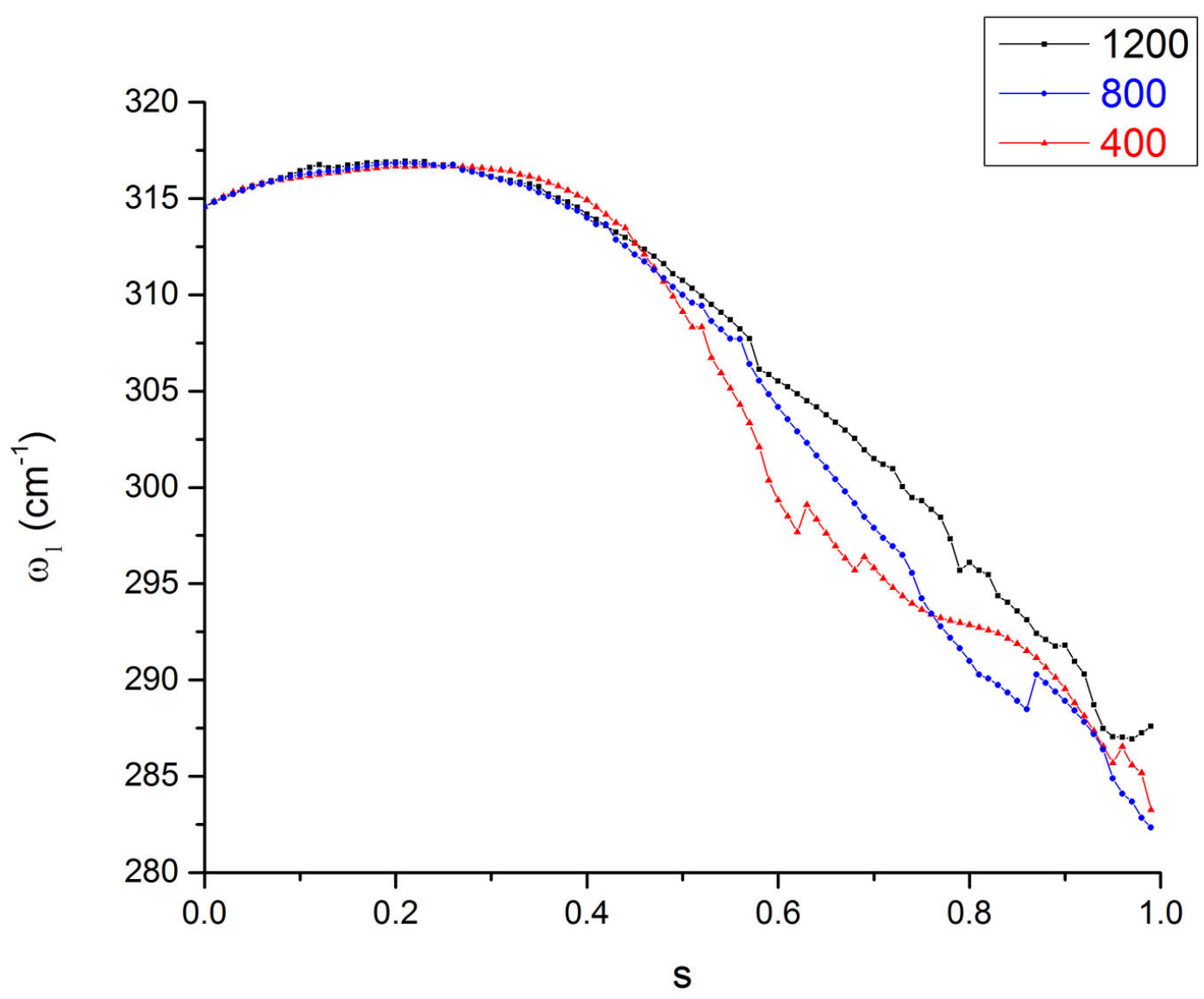

(a) $\mathrm{Li}_{3}$ fit values for $\omega_{1}$.

Figure 2: Panels (a-h) show fitted parameter values as a function of $s$ for the $\mathrm{Li}_{3}$ potential. Red line indicates that levels fit are truncated at $400 \mathrm{~cm}^{-1}$. Blue line indicates that levels fit are truncated at $800 \mathrm{~cm}^{-1}$. Black line indicates that levels fit are truncated at $1200 \mathrm{~cm}^{-1}$. 


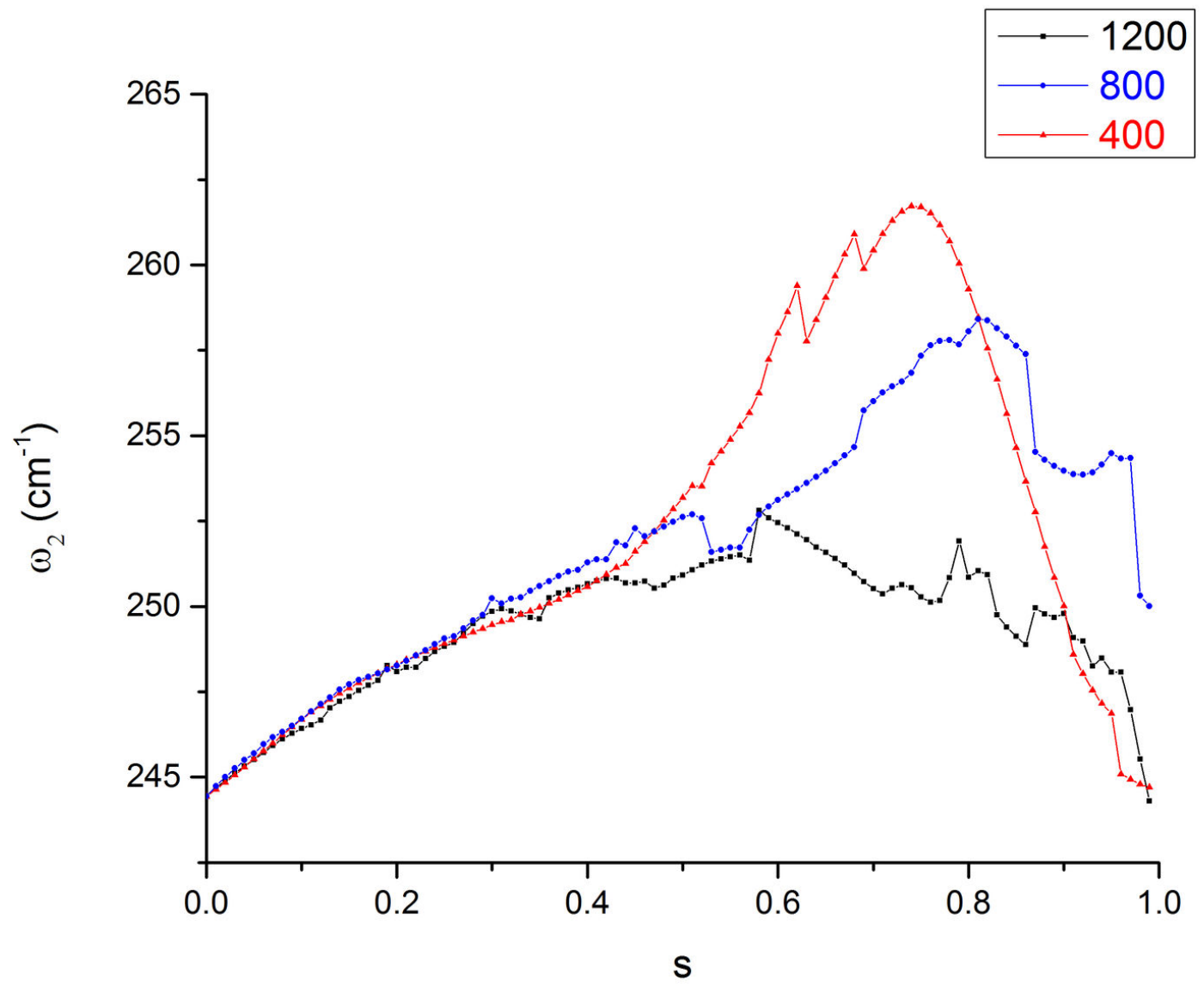

(b) $\mathrm{Li}_{3}$ fit values for $\omega_{2}$. 


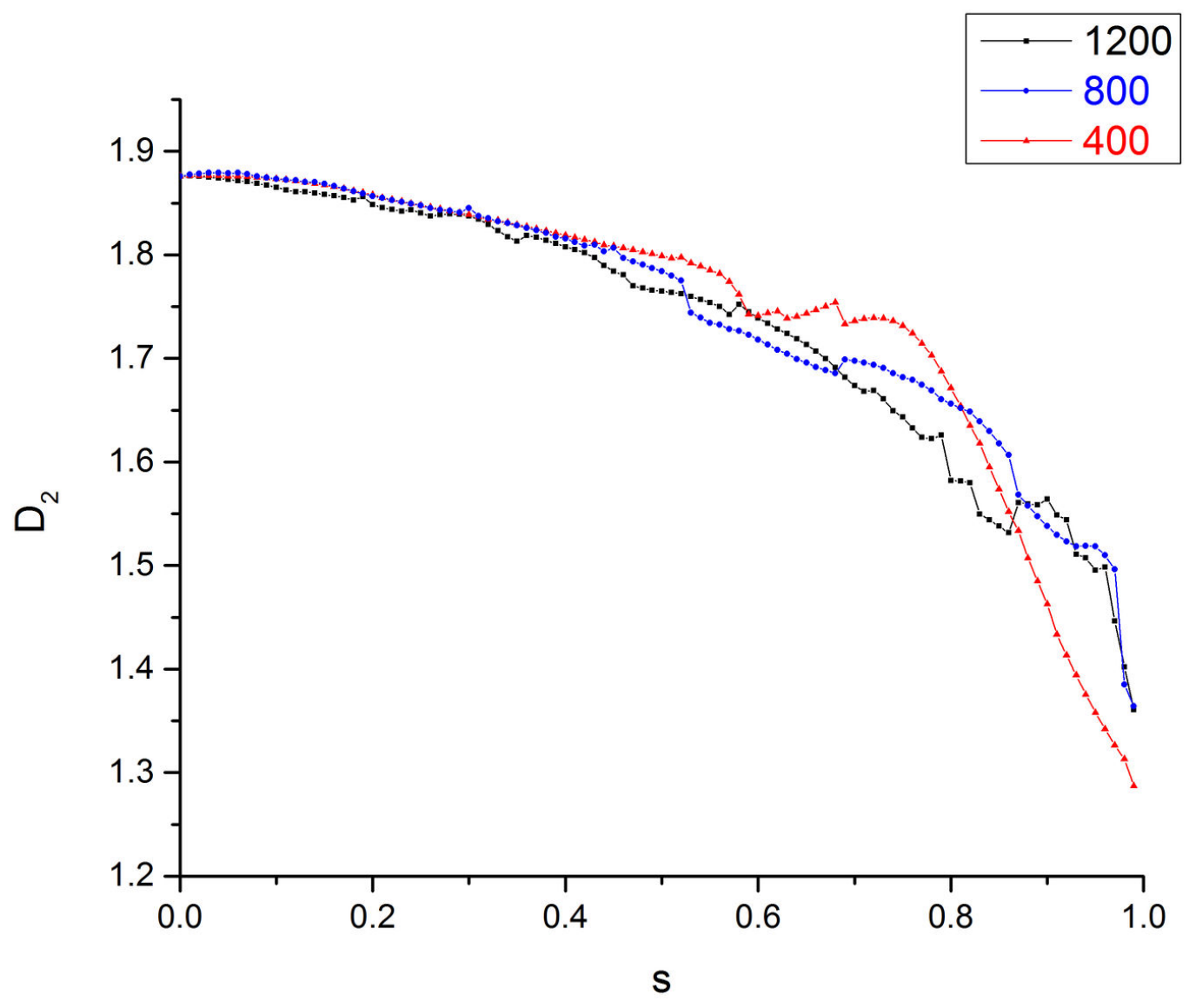

(c) $\mathrm{Li}_{3}$ fit values for $D_{2}$. 


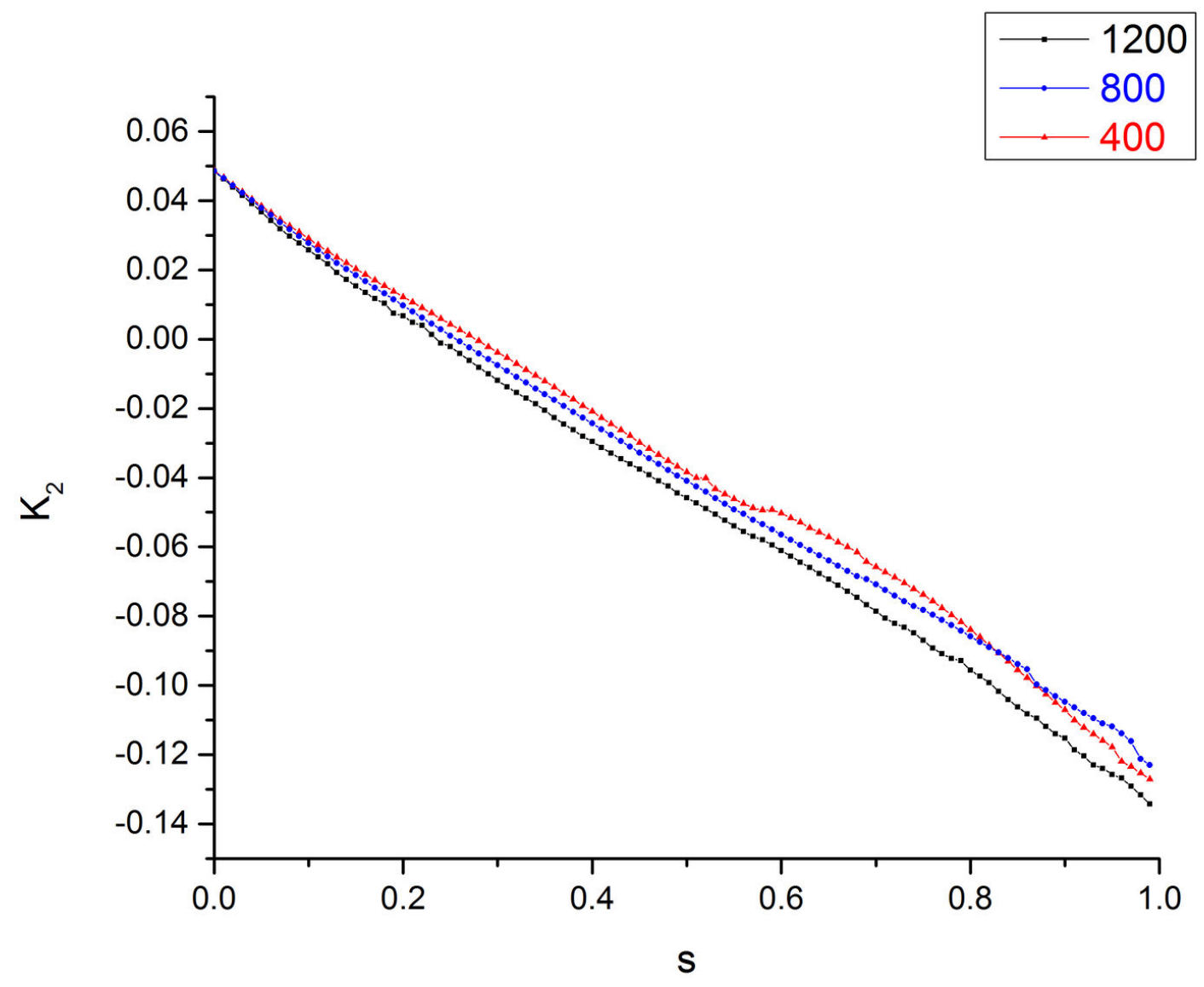

(d) $\mathrm{Li}_{3}$ fit values for $K_{2}$. 


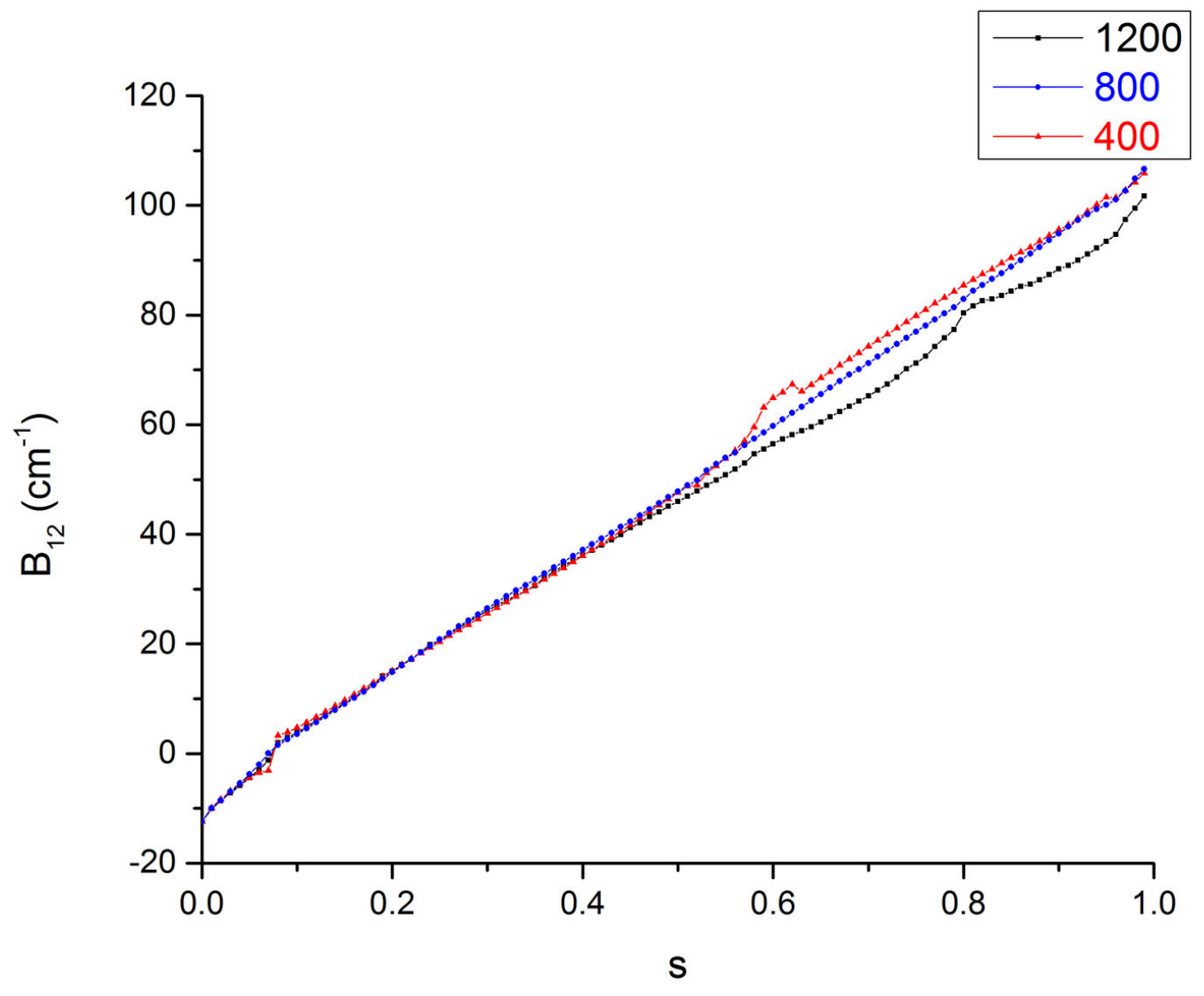

(e) $\mathrm{Li}_{3}$ fit values for $B_{12}$. 


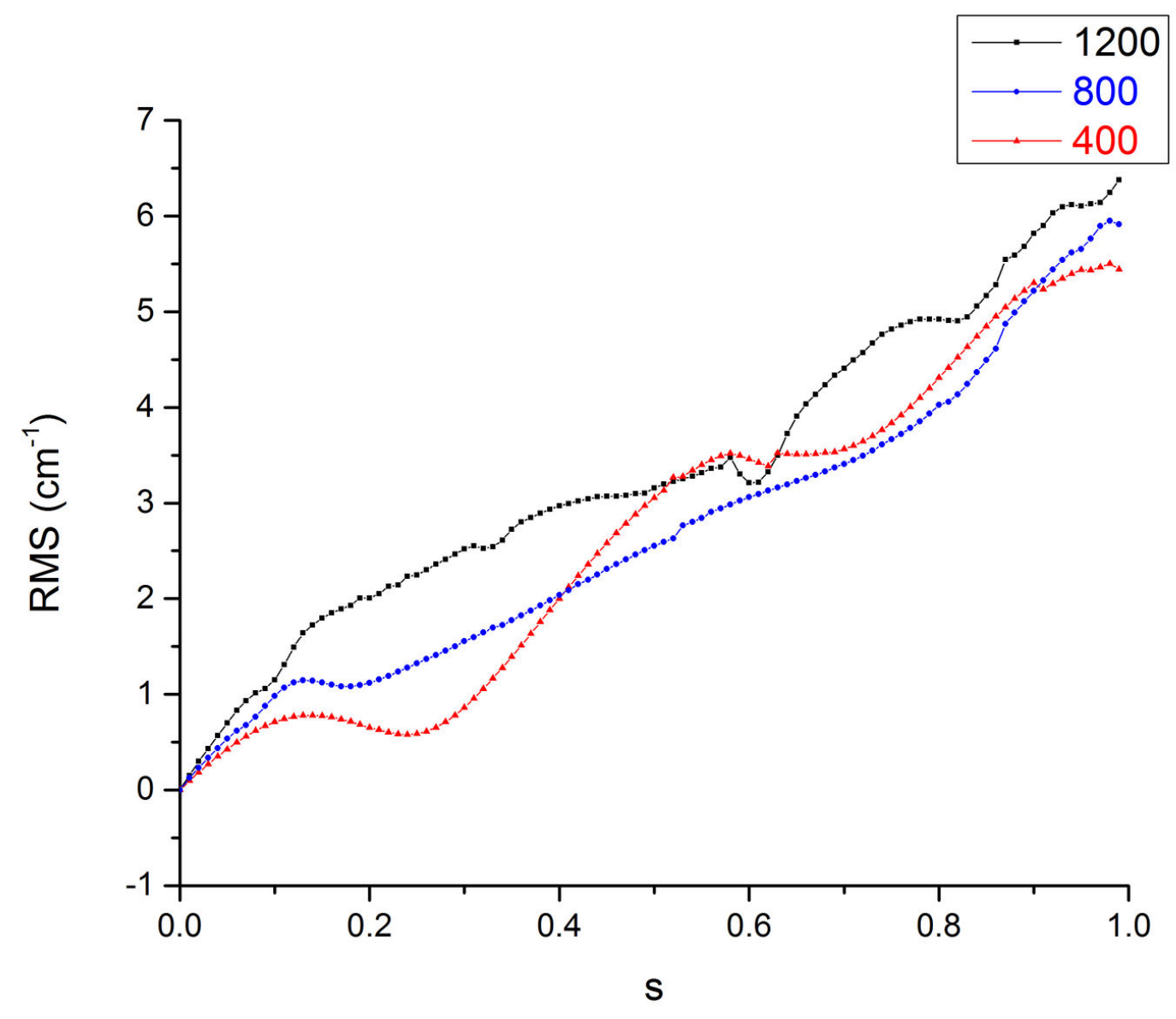

(f) $\mathrm{Li}_{3} \mathrm{RMS}$ values of fits. 


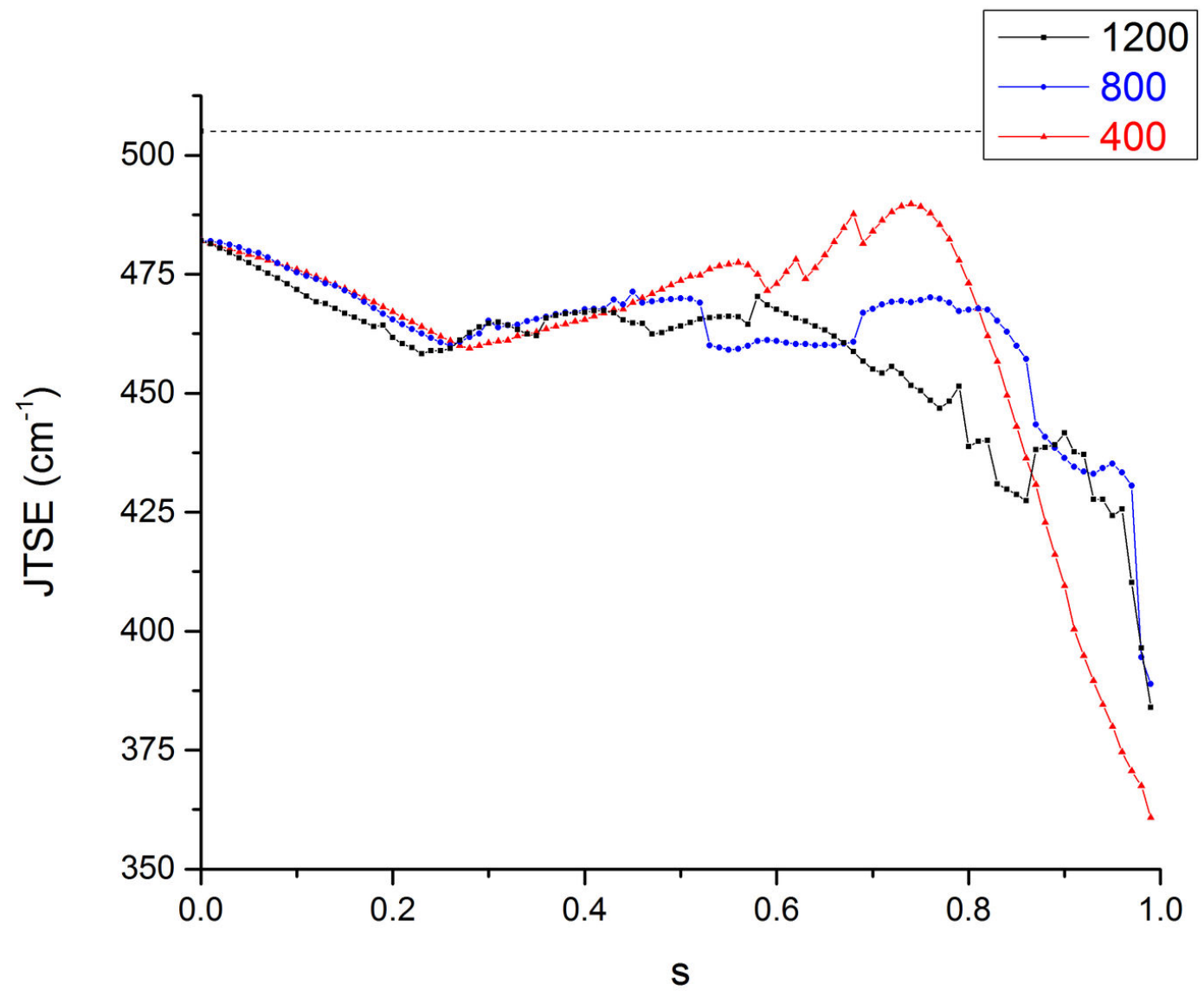

(g) $\mathrm{Li}_{3} \mathrm{JTSE}$ values of fits. The true value of $505 \mathrm{~cm}^{-1}$ is marked with the dotted line. 


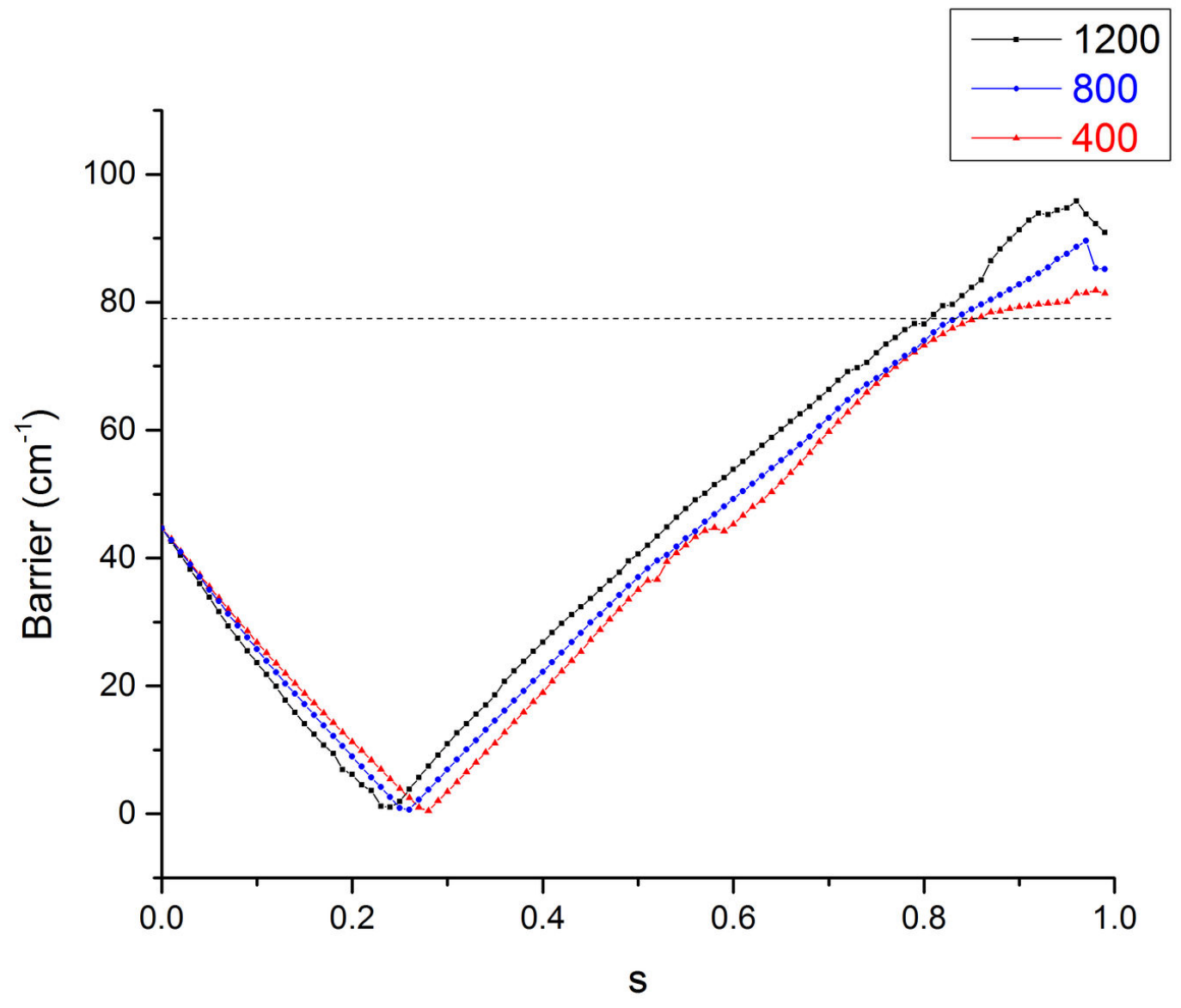

(h) $\mathrm{Li}_{3}$ pseudo-rotation barrier values of fits. The true value of $77.5 \mathrm{~cm}^{-1}$ is marked with the dotted line. 


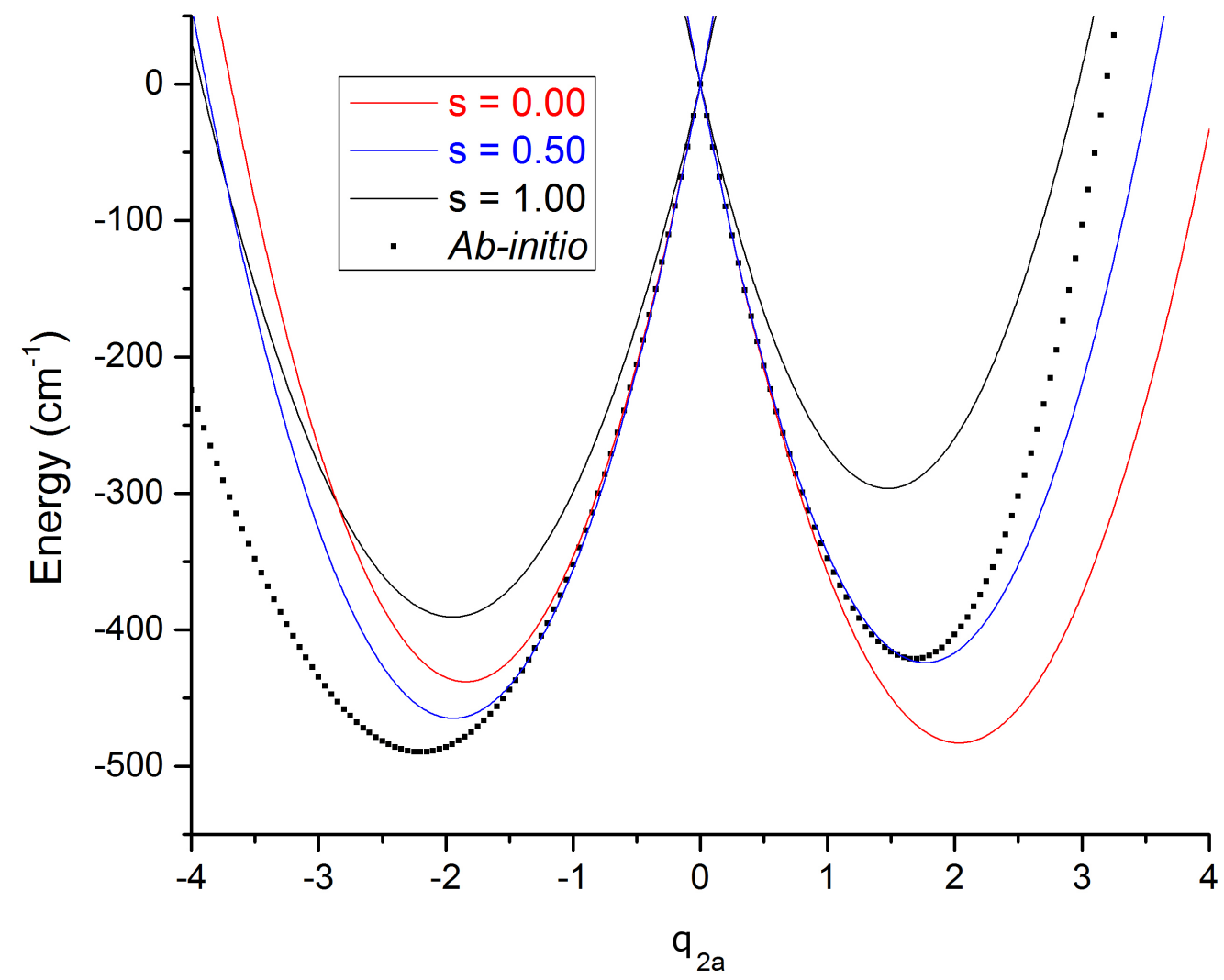

Figure 3: The PES along the $a_{2 a}$ coordinate for the quadratic fit at $s=0.00$ (red), $s=0.50$ (blue), and 1.00 (black). The dotted line is the ab initio calculation. 


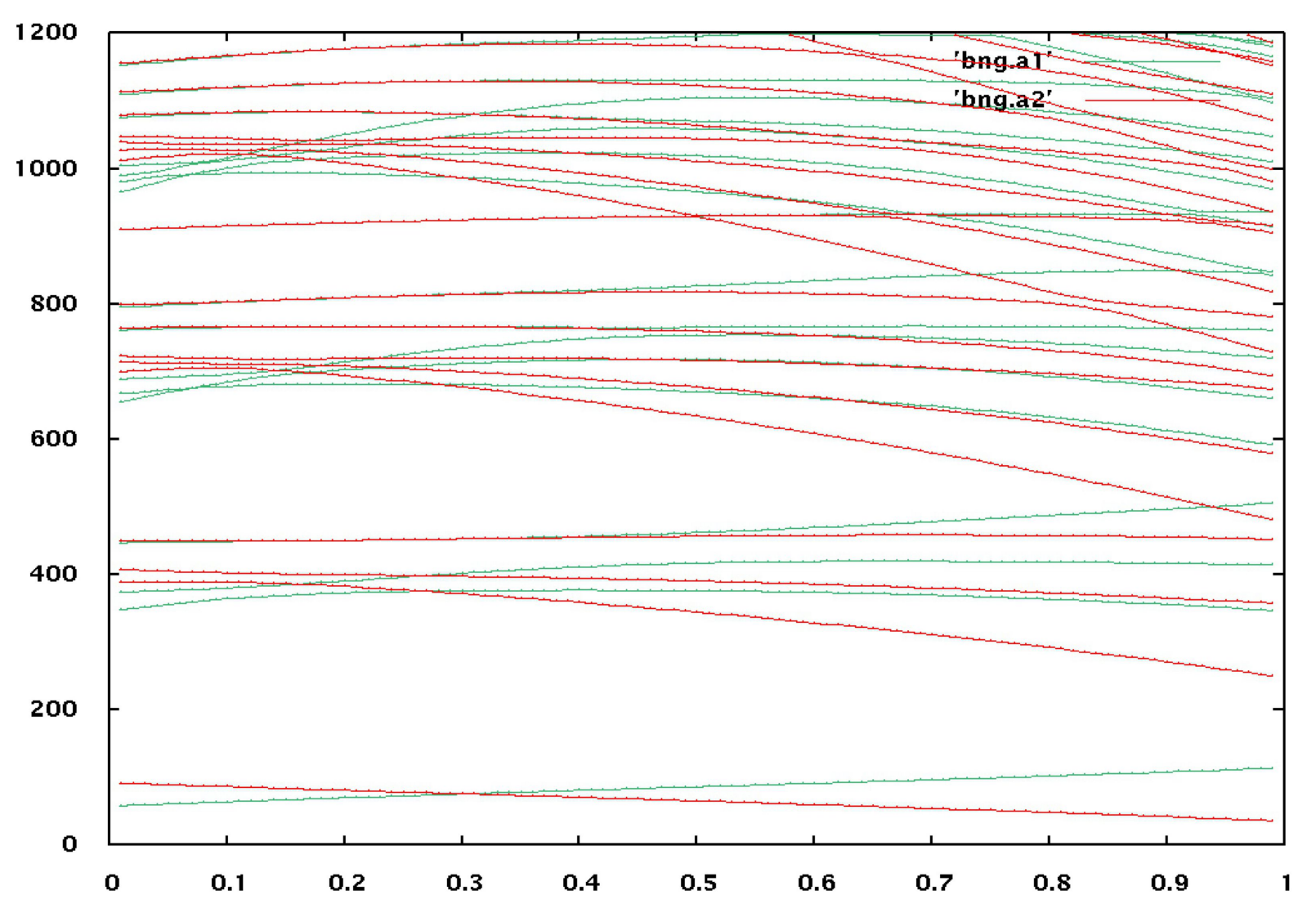

Figure 4: The energies of the vibronic levels with $a_{1}^{\prime}$ vibronic symmetry (green) and $a_{2}^{\prime}$ vibronic symmetry (red) as $s$ is increased. A crossing between these two sets of levels is seen between $s=0.2$ and $s=0.25$. 


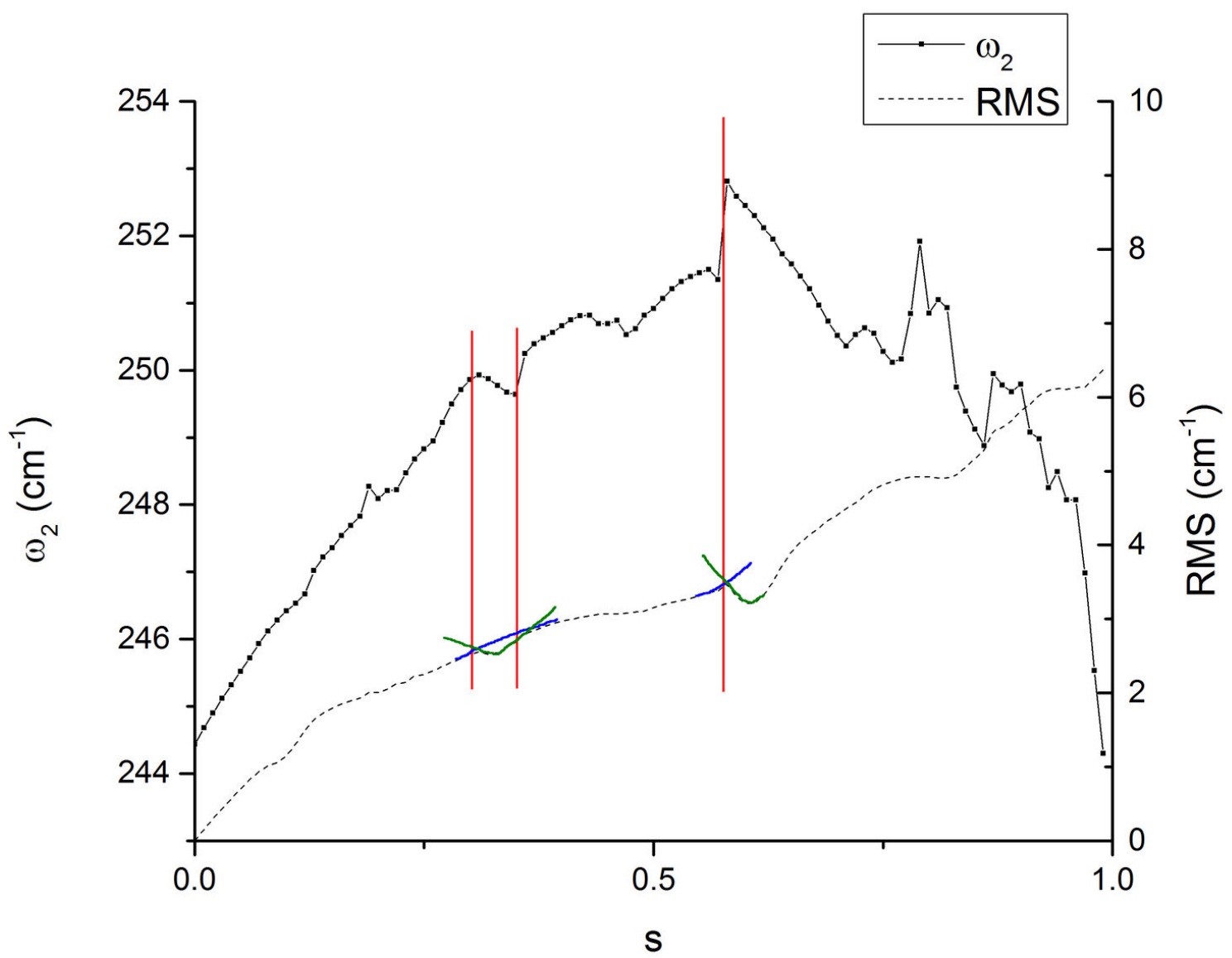

Figure 5: The plot of $\omega_{2}$ (black, full line) and RMS (black, dashed line) are plotted against $s$. Vertical lines mark regions where the plot of $\omega_{2}$ changes abruptly. A corresponding jagged change in the RMS plot is also seen at the marked region, indicating a jump to a different RMS minimum. Blue and green lines highlight the shape of the two different RMS minimum between which the fit is switching. 
Table 1: Relationships governing linear, quadratic, cubic and quartic force constants involving the totally symmetric mode $\left(q_{1}\right)$ and the $a_{1}$ component of the degenerate mode $\left(q_{2 a}\right)$ associated with the potential energy surfaces of the ${ }^{2} A_{1}$ and ${ }^{2} B_{2}$ adiabatic sublevels of the ${ }^{2} E^{\prime}$ electronic state of $\mathrm{Li}_{3}$ to parameters of the potential energy function in the diabatic Jahn-Teller basis (Eq. (6)). The individual columns of the table refer to the electronic block of the diabatic potential, where $A$ and $B$ are the $a_{1}$ and $b_{2}$ components of the degenerate state.

\begin{tabular}{|c|c|c|c|}
\hline$\overline{\text { Electronic Block }}$ & $\mathrm{AA}$ & $\overline{\mathrm{BB}}$ & $\overline{\mathrm{AB}}$ \\
\hline$F_{1}$ & 0 & 0 & \\
\hline$F_{2 a}$ & $f_{2 a}^{A}$ & $-f_{2 a}^{A}$ & 0 \\
\hline$F_{2 b}$ & 0 & 0 & $f_{2 a}^{A}$ \\
\hline$F_{11}$ & $f_{11}^{A}$ & $f_{11}^{A}$ & 0 \\
\hline$F_{12 a}$ & $f_{12 a}^{A}$ & $-f_{12 a}^{A}$ & 0 \\
\hline$F_{12 b}$ & 0 & 0 & $f_{12 a}^{A}$ \\
\hline$F_{2 a 2 a}$ & $f_{2 a 2 a}^{A}$ & $f_{2 a 2 a}^{B}$ & 0 \\
\hline$F_{2 b 2 b}$ & $f_{2 a 2 a}^{B}$ & $f_{2 a 2 a}^{A}$ & 0 \\
\hline$F_{2 a 2 b}$ & 0 & 0 & $\frac{1}{2}\left(f_{2 a 2 a}^{B}-f_{2 a 2 a}^{A}\right)$ \\
\hline$F_{111}$ & $f_{111}^{A}$ & $f_{111}^{A}$ & 0 \\
\hline$F_{112 a}$ & $f_{112 a}^{A}$ & $-f_{112 a}^{A}$ & 0 \\
\hline$F_{112 b}$ & 0 & 0 & $f_{112 a}^{A}$ \\
\hline$F_{12 a 2 a}$ & $f_{12 a 2 a}^{A}$ & $f_{12 a 2 a}^{B}$ & 0 \\
\hline$F_{12 b 2 b}$ & $f_{12 a 2 a}^{B}$ & $f_{12 a 2 a}^{A}$ & 0 \\
\hline$F_{12 a 2 b}$ & 0 & 0 & $\frac{1}{2}\left(f_{12 a 2 a}^{B}-f_{12 a 2 a}^{A}\right)$ \\
\hline$F_{2 a 2 a 2 a}$ & $f_{2 a 2 a 2 a}^{A}$ & $f_{2 a 2 a 2 a}^{B}$ & 0 \\
\hline$F_{2 a 2 a 2 b}$ & 0 & 0 & $\frac{1}{6}\left(f_{2 a 2 a 2 a}^{A}-f^{B} 2 a 2 a 2 a\right)$ \\
\hline$F_{2 a 2 b 2 b}$ & $-\frac{1}{3}\left(f_{2 a 2 a 2 a}^{A}+2 f_{2 a 2 a 2 a}^{B}\right)$ & $-\frac{1}{3}\left(2 f_{2 a 2 a 2 a}^{A}+f_{2 a 2 a 2 a}^{B}\right)$ & 0 \\
\hline$F_{2 b 2 b 2 b}$ & 0 & 0 & $\frac{1}{2}\left(f_{2 a 2 a 2 a}^{A}-f_{2 a 2 a 2 a}^{B}\right)$ \\
\hline$F_{1111}$ & $f_{1111}^{A}$ & $f_{1111}^{A}$ & 0 \\
\hline$F_{1112 a}$ & $f_{1112 a}^{A}$ & $-f_{1112 a}^{A}$ & 0 \\
\hline$F_{1112 b}$ & 0 & 0 & $f_{1112 a}^{A}$ \\
\hline$F_{112 a 2 a}$ & $f_{112 a 2 a}^{A}$ & $f_{112 a 2 a}^{B}$ & 0 \\
\hline$F_{112 b 2 b}$ & $f_{112 a 2 a}^{B}$ & $f_{112 a 2 a}^{A}$ & 0 \\
\hline$F_{112 a 2 b}$ & 0 & 0 & $\frac{1}{2}\left(f_{112 a 2 a}^{B}-f_{112 a 2 a}^{A}\right)$ \\
\hline$F_{12 a 2 a 2 a}$ & $f_{12 a 2 a 2 a}^{A}$ & $f_{12 a 2 a 2 a}^{B}$ & 0 \\
\hline$F_{12 a 2 a 2 b}$ & 0 & 0 & $\frac{1}{6}\left(f_{12 a 2 a 2 a}^{A}-f^{B} 12 a 2 a 2 a\right)$ \\
\hline$F_{12 a 2 b 2 b}$ & $-\frac{1}{3}\left(f_{12 a 2 a 2 a}^{A}+2 f_{12 a 2 a 2 a}^{B}\right)$ & $-\frac{1}{3}\left(2 f_{12 a 2 a 2 a}^{A}+f_{12 a 2 a 2 a}^{B}\right)$ & 0 \\
\hline$F_{12 b 2 b 2 b}$ & 0 & 0 & $\frac{1}{2}\left(f_{12 a 2 a 2 a}^{A}-f_{12 a 2 a 2 a}^{B}\right)$ \\
\hline
\end{tabular}


Table 2: Quadratic parameters for the $\tilde{X}^{2} E^{\prime}$ state of $\mathrm{Li}_{3}$ based on the original analysis[78] of the experimental data and alternative fits removing questionable levels. Refit I is a fit using the quadratic JT Hamiltonian with the level at $66 \mathrm{~cm}^{-1}$ removed. Refit II is a fit using the quadratic JT Hamiltonian with both the level at $66 \mathrm{~cm}^{-1}$ and $205 \mathrm{~cm}^{-1}$ are excluded. The parameter $B_{12}$ was not fit in Wöste's analysis.

\begin{tabular}{lrrr}
\hline \hline Parameter & Exp. fit[78] & Refit I & Refit II \\
\hline$\omega_{1}\left(\mathrm{~cm}^{-1}\right)$ & 301 & 223.81 & 283.31 \\
$\omega_{2}\left(\mathrm{~cm}^{-1}\right)$ & 150 & 206.39 & 202.48 \\
$D_{2}$ & 1.6928 & 1.2245 & 1.5840 \\
$K_{2}$ & 0.0500 & -0.1381 & -0.1701 \\
$B_{12}\left(\mathrm{~cm}^{-1}\right)$ & 0 & 68.9 & 0 \\
JTSE $\left(\mathrm{cm}^{-1}\right)$ & 267 & 293 & 386 \\
Barrier $\left(\mathrm{cm}^{-1}\right)$ & 25.5 & 71.2 & 112 \\
\hline \hline
\end{tabular}


Table 3: Assignments of the $\operatorname{Li}_{3} \tilde{X}^{2} E^{\prime}$ state vibronic levels from various analyses. Experimental levels were obtained and fit by Dugourd, et al.[78] using a quadratic JT Hamiltonian. Refit I and Refit II are obtained by fitting using a quadratic JT Hamiltonian after removing questionable levels in the data set. The $4^{\text {th }}$ Order Calc. is obtained from the quartic JT Hamiltonian using derivatives calculated from the ab initio PES. All units are $\mathrm{cm}^{-1}$.

\begin{tabular}{l|lll|l}
\hline \hline $\operatorname{Exp}[78]$ & Ref. [78] & Refit I & Refit II & $4^{\text {th }}$ Order Calc. \\
\hline 37 & $38 / a_{2}^{\prime}$ & $37 / a_{2}^{\prime}$ & $37 / a_{2}^{\prime}$ & $35 / a_{2}^{\prime}$ \\
$66^{a}$ & $60 / a_{1}^{\prime}$ & $-/-$ & $-/-$ & $-/-$ \\
115 & $117 / e^{\prime}$ & $115 / a_{1}^{\prime}$ & $123 / a_{1}^{\prime}$ & $114 / a_{1}^{\prime}$ \\
164 & $164 / e^{\prime}$ & $152 / e^{\prime}$ & $156 / e^{\prime}$ & $155 / e^{\prime}$ \\
205 & $201 / e^{\prime}$ & $202 e^{\prime}$ & $-/-$ & $-/-$ \\
227 & $220 / a_{2}^{\prime}$ & $230 / a_{2}^{\prime}$ & $218 / e^{\prime}$ & $225 / e^{\prime}$ \\
245 & $246 / a_{1}^{\prime}$ & $246 / e^{\prime}$ & $250 / a_{2}^{\prime}$ & $247 / a_{2}^{\prime}$ \\
290 & $287 / a_{2}^{\prime}$ & $293 / a_{2}^{\prime}$ & $283 / e^{\prime}$ & $300 / e^{\prime}$ \\
303 & $-/-$ & $309 / e^{\prime}$ & $313 / a_{2}^{\prime}$ & $345 / a_{1}^{\prime}$ \\
313 & $310 / e^{\prime}$ & $313 / a_{1}^{\prime}$ & $315 / e^{\prime}$ & $355 / e^{\prime}$ \\
339 & $344 / e^{\prime}$ & $341 / e^{\prime}$ & $339 / a_{1}^{\prime}$ & $356 / a_{2}^{\prime}$ \\
372 & $381 / e^{\prime}$ & $365 / a_{1}^{\prime}$ & $374 / e^{\prime}$ & $370 / e^{\prime}$ \\
410 & $408 / e^{\prime}$ & $406 / e^{\prime}$ & $406 / a_{1}^{\prime}$ & $-/-$ \\
\hline RMS & 4.45 & 4.62 & 6.52 & \\
\hline \hline
\end{tabular}

${ }^{a}$ Not reported by Kramer et al.[79] 
Table 4: Vibrational frequencies $(\omega)$ and derivatives $(F)$ of the $\mathrm{Li}_{3}$ potential for Equation (17). All units are $\mathrm{cm}^{-1}$.

\begin{tabular}{ll}
\hline \hline$\omega_{1}$ & 314.5757 \\
$\omega_{2}$ & 244.4367 \\
$F_{2 a}^{11}$ & -473.4924 \\
$F_{2 b}^{12}$ & -473.4924 \\
$F_{2 a}^{22}$ & 473.4924 \\
$F_{12 a}^{11}$ & -11.5894 \\
$F_{2 a 2 a}^{11}$ & 232.5129 \\
$F_{2 b 2 b}^{11}$ & 256.3604 \\
$F_{12 b}^{12}$ & -11.5894 \\
$F_{2 a 2 b}^{12}$ & 11.9237 \\
$F_{12 a}^{22}$ & 11.5894 \\
$F_{2 a 2 a}^{22}$ & 256.3604 \\
$F_{2 b 3 b}^{22}$ & 232.5129 \\
$F_{111}^{11}$ & 85.9739 \\
$F_{112 a}^{11}$ & -0.8209 \\
$F_{12 a 2 a}^{11}$ & 49.9472 \\
$F_{12 b 2 b}^{11}$ & 56.3025 \\
$F_{2 a 2 a 2 a}^{11}$ & 55.692 \\
$F_{2 a 2 b 2 b}^{11}$ & -46.4393 \\
$F_{112 b}^{12}$ & -0.8209 \\
$F_{12 a 2 b}^{12}$ & 3.1776 \\
$F_{2 a 2 a 2 b}^{12}$ & 2.3131 \\
$F_{2 b 2 b 2 b}^{12}$ & 6.9395 \\
\hline & 85.9739 \\
\hline
\end{tabular}

Continued 


\begin{tabular}{ll} 
Table $4:$ & Continued \\
\hline$F_{112 a}^{22}$ & 0.8209 \\
$F_{12 a 2 a}^{22}$ & 56.3025 \\
$F_{12 b 2 b}^{22}$ & 49.9472 \\
$F_{2 a 2 a 2 a}^{22}$ & 41.8129 \\
$F_{2 a 2 b 2 b}^{22}$ & -51.0656 \\
$F_{1111}^{11}$ & 19.4109 \\
$F_{1112 a}^{11}$ & -0.089 \\
$F_{112 a 2 a}^{11}$ & 7.0757 \\
$F_{112 b 2 b}^{11}$ & 10.9193 \\
$F_{12 a 2 a 2 a}^{11}$ & 13.8875 \\
$F_{12 a 2 b 2 b}^{11}$ & -12.2873 \\
$F_{2 a 2 a 2 a 2 a}^{11}$ & 6.0571 \\
$F_{2 a 2 a 2 b 2 b}^{11}$ & 2.019 \\
$F_{2 b 2 b 2 b 2 b}^{11}$ & 6.0571 \\
$F_{1112 b}^{12}$ & -0.089 \\
$F_{112 a 2 b}^{12}$ & 1.9218 \\
$F_{12 a 2 a 2 b}^{12}$ & 0.4 \\
$F_{12 b 2 b 2 b}^{12}$ & 1.2001 \\
$F_{1111}^{22}$ & 19.4109 \\
$F_{1112 a}^{22}$ & 0.089 \\
$F_{112 a 2 a}^{22}$ & 10.9193 \\
$F_{112 b 2 b}^{22}$ & 7.0757 \\
$F_{12 a 2 a 2 a}^{22}$ & 11.4872 \\
$F_{12 a 2 b 2 b}^{22}$ & -13.0874 \\
\hline $2 a 2 a 2 a 2 a$ & 6.0571 \\
\hline & \\
\hline
\end{tabular}

Continued 
Table 4: Continued

\begin{tabular}{ll}
$F_{2 a 2 a 2 b 2 b}^{22}$ & 2.019 \\
$F_{2 b 2 b 2 b 2 b}^{22}$ & 6.0571 \\
\hline \hline
\end{tabular}


Table 5: Vibronic energy levels calculated using the quartic JT Hamiltonian parametrized by the values in Table 4. The column JT2 zeros the cubic and quartic derivatives, JT4 utilizes all the derivatives.

\begin{tabular}{llc}
\hline \hline JT4 & JT2 & Vibronic Symmetry \\
\hline 35 & 91.8 & $a_{2}^{\prime}$ \\
114 & 57.2 & $a_{1}^{\prime}$ \\
155 & 179.2 & $e^{\prime}$ \\
225 & 261.2 & $e^{\prime}$ \\
247 & 388.4 & $a_{2}^{\prime}$ \\
300 & 311.6 & $e^{\prime}$ \\
345 & 346.5 & $a_{1}^{\prime}$ \\
355 & 316 & $e^{\prime}$ \\
356 & 407.6 & $a_{2}^{\prime}$ \\
370 & 486.4 & $e^{\prime}$ \\
\hline \hline
\end{tabular}




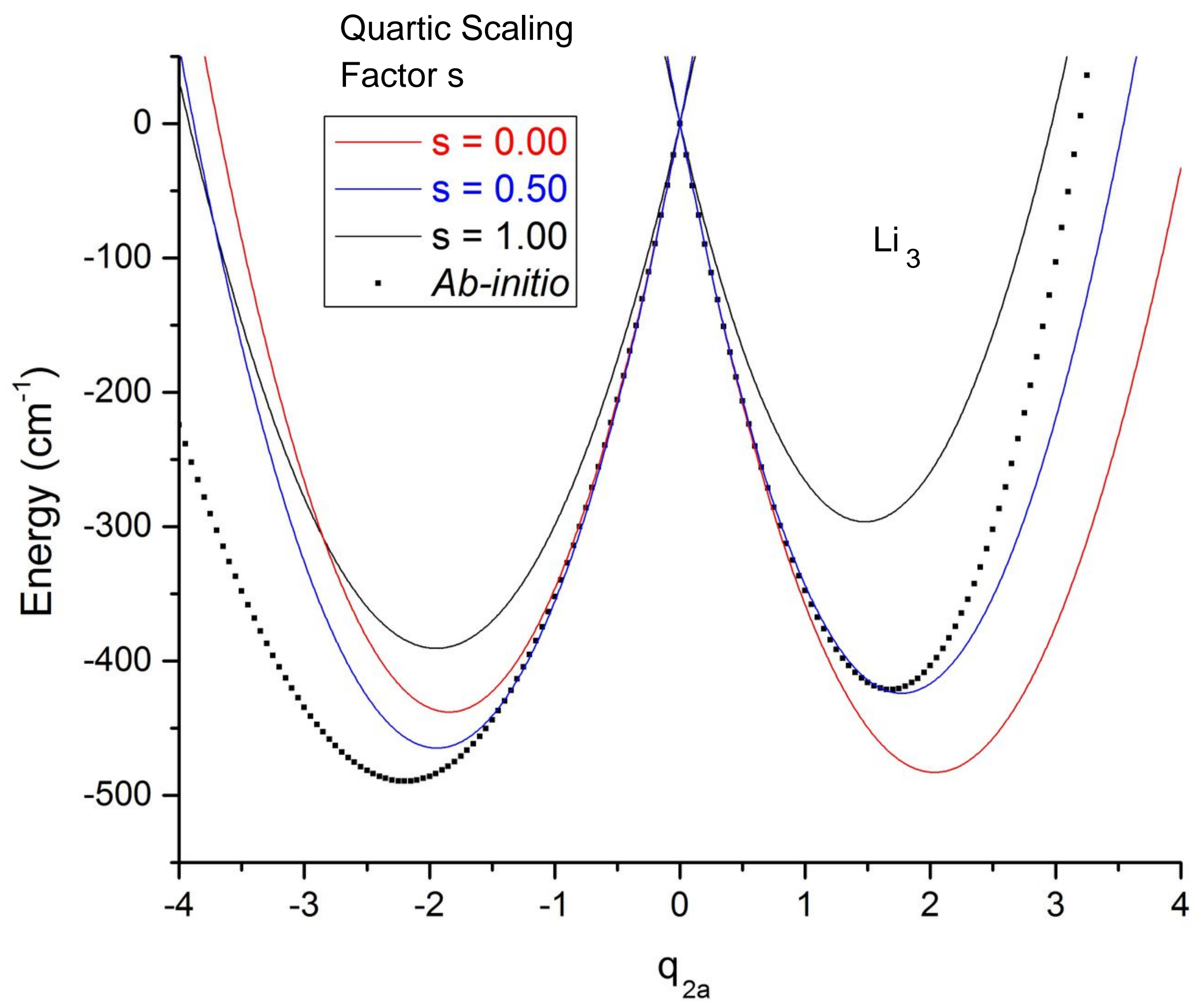

